\title{
Multilocus phylogeny and ecological differentiation of the "Eupelmus urozonus species group" (Hymenoptera, Eupelmidae) in the West-Palaearctic
}

\author{
F. Al khatib ${ }^{1,3^{*}}$, A. Cruaud ${ }^{2}$, L. Fusu ${ }^{4}$, G. Genson ${ }^{2}$, J.-Y. Rasplus ${ }^{2}$, N. Ris ${ }^{1}$ and G. Delvare ${ }^{3}$
}

\begin{abstract}
Background: The ecological differentiation of insects with parasitic life-style is a complex process that may involve phylogenetic constraints as well as morphological and/or behavioural adaptations. In most cases, the relative importance of these driving forces remains unexplored. We investigate here this question for the "Eupelmus urozonus species group" which encompasses parasitoid wasps of potential interest in biological control. This was achieved using seven molecular markers, reliable records on 91 host species and a proxy of the ovipositor length.
\end{abstract}

Results: After using an adequate partitioning scheme, Maximum likelihood and Bayesian approaches provide a well-resolved phylogeny supporting the monophyly of this species group and highlighting its subdivision into three sub-groups. Great variations of both the ovipositor length and the host range (specialist versus generalist) were observed at this scale, with these two features being not significantly constrained by the phylogeny. Ovipositor length was not shown as a significant predictor of the parasitoid host range.

Conclusions: This study provides firstly the first evidence for the strong lability of both the ovipositor's length and the realised host range in a set of phylogenetically related and sympatric species. In both cases, strong contrasts were observed between sister species. Moreover, no significant correlation was found between these two features. Alternative drivers of the ecological differentiation such as interspecific interactions are proposed and the consequences on the recruitment of these parasitoids on native and exotic pests are discussed.

Keywords: Ecological specialization, Ectoparasitoid, Host range evolution, Molecular phylogeny, Morphological adaptation, Ovipositor, Phylogenetic constraint

\section{Background}

Ecological speciation is a process in which polymorphism within populations (e.g. in resource use or habitat preference) ultimately induces the appearance of two sister species, each adapted to a different niche [1-4]. According to Rundle and Nosil [2], three principal components must be involved: i) a source of divergent selection, ii) a form of reproductive isolation, and iii) a genetic

\footnotetext{
* Correspondence: fadel.alkhatib@sophia.inra.fr

'INRA, University of Nice Sophia Antipolis, CNRS, UMR 1355-7254 Institut Sophia Agrobiotech, Sophia Antipolis 06900, France

${ }^{3}$ CIRAD, UMR 55 CBGP, 755 Avenue du Campus Agropolis, CS 30016 F-34988, Montferrier-sur-Lez Cedex, France

Full list of author information is available at the end of the article
}

mechanism linking divergent selection to reproductive isolation. Among plant-feeding insects, several empirical studies support this scenario $[1,5,6]$, which can also occur for insects with a parasitic lifestyle, in particular within the upper trophic levels. For such organisms, ecological differentiation between sister species can also be driven by the ecological differentiation of their hosts via a process called sequential or cascading speciation [7-9]. If pervasive enough, such processes should lead to the clustering of phylogenetically related specialists.

Additionally, transitions between generalists to specialists (and vice-versa) are also occurring and, so far, empirical data provide a mixed picture about the 
relative frequencies of evolution toward specialisation and generalization [10-13]. However, transitions from generalist ancestors to specialized species are probably recurrent as (i) generalist species are unlikely to produce "jack-of-all trades-master of none" genotypes because of genetic or physiological trade-off [14-16]; (ii) the subsequent acquisition of specialized genotypes may be a primary step towards speciation [17-19]; and (iii) specialist species may be more prone to extinction $[13,20]$. At a phylogenetic level, both kinds of transitions should lead to the mixing of both specialists and generalists within the same cluster.

Questions of (i) the host range (specialist versus generalist) of ancestral species of current specialists and (ii) the distribution of host ranges within a phylogeny were recently addressed by Hardy and Otto [21]. They illustrated them using two notions, respectively "the musical chairs hypothesis" (specialists originate from specialists through host switch) and the "oscillation hypothesis" (specialists originate from generalists, with some specialists widening their host range before the next speciation event). The extent to which one of these scenarios is more frequent has nevertheless still to be evaluated rigorously for the organisms with a parasitic lifestyle.

Parasitoids are organisms (mainly Hymenoptera and Diptera) whose pre-imaginal life depends on the successful exploitation of a single host [22, 23]. Behind this simple definition, a great diversity of life history strategies and physiological adaptations are observed. In particular, the ovipositor allows egg-laying by the female and is thus a key organ especially for species that are exploiting concealed or protected hosts [24, 25]. The features (in particular the length) of this organ and its ability to evolve could contribute to drive specialization and/or speciation. Focusing on the "Eupelmus urozonus species group" (Hymenoptera: Eupelmidae), we examine here whether the host range is subject to phylogenetic constraints and/or whether the ovipositor length is a significant driver of host use.

Within the subfamily Eupelminae (33 genera), the genus Eupelmus Dalman is the most diverse, with 91 available valid species names in the Palaearctic region [26]. Species of Eupelmus are primary or facultative secondary ectoparasitoids whose larvae develop as idiobionts on the immature stages (larvae, pupae and more rarely eggs) of many insects (beetles, flies, moths, wasps or cicadas) that are concealed or protected in plant tissues (stems, galls, fruits or seeds) [27]. Most Eupelmus are considered as generalist parasitoids [27, 28]. However, because of both the extreme sexual dimorphism characterizing the subfamily and the existence of species groups possibly hiding cryptic species, the systematics and the evolutionary ecology of these species remain poorly understood. This situation is well illustrated with the "E. urozonus species complex/group" which was repeatedly investigated $[27,29-31]$ until its recent revision within the Palaearctic region by Al khatib et al. [32, 33], which identified 11 new species in this region. Semantically, the term "complex" used in Al khatib et al. [32, 33] is substituted here by the term "species group" (Al khatib et al. in preparation). As a consequence of this unsuspected biodiversity, most of the published host records for these species are unreliable because all of the common species with a comparatively short ovipositor (E. gemellus Al khatib, 2015, E. confusus Al khatib, 2015, and especially $E$. kiefferi De Stefani, 1898) were misidentified as E. urozonus Dalman, 1820, while the two common species with a comparatively long ovipositor (E. azureus Ratzeburg, 1844 and $E$. annulatus Nees, 1834) were both frequently mistreated under E. annulatus [29, 34].

In the present study, we first provide a reliable molecular phylogeny of the "E. urozonus species group" using a multi-locus approach. Then, for most of the species, we compile host records and data on ovipositor length. We finally carry out a comparative analysis to evaluate the role of phylogenetic constraints in the evolution of ovipositor length and host range as well as the role of the ovipositor's length in determining the host range.

\section{Methods}

\section{Sampling}

A total of 31 species, with 91 individuals, sampled in the Palaearctic region were included in this study.

- Eighteen of the 21 species within the "urozonus species group" that were recently revised using both morphological and molecular characters [32, 33]: $E$. acinellus Askew, 2009, E. annulatus, E. azureus, E. cerris Förster, 1860, E. confusus, E. fulvipes Förster, 1860, E. gemellus, E. janstai Delvare and Gibson, 2015, E. kiefferi, E. longicalvus Al khatib \& Fusu, 2015, E. minozonus Delvare, 2015, E. opacus Delvare, 2015, E. pistaciae Al khatib, 2015, E. priotoni Delvare, 2015, E. purpuricollis Fusu \& Al khatib, 2015, E. simizonus Al khatib, 2015, E. tibicinis Bouček, 1963 and E. urozonus.

- Thirteen species were used as outgroup including (i) species belonging to the three subgenera of Eupelmus sensu Gibson (1995): Eupelmus [E. atropurpureus Dalman, 1820, E. matranus Erdős, 1947, E. microzonus Förster, 1860, E. pini Taylor, 1927 and E. vindex Erdős, 1955]; Macroneura Walker [E. falcatus (Nikol'skaya, 1952) and $E$. seculatus Kalina, 1981], and Episolindelia Girault [E. linearis Förster, 1860, E. testaceiventris (Motschulsky, 1863) and E. juniperinus thuriferae Askew, 2000]; and (ii) species belonging to other 
genera within Eupelminae, Reikosiella (Hirticauda) [R. aff. rostrata (Ruschka, 1921)] and Anastatus Motschulsky [Anastatus sidereus (Erdős, 1957) and Anastatus aff. temporalis Askew, 2005]. The species were identified by the authors using the available identification keys [29, 31, 35-37].

Specimens were killed with ethyl acetate and preserved in $95 \%$ ethanol at $-20{ }^{\circ} \mathrm{C}$ until DNA extraction. After the DNA extraction, the voucher specimens were prepared as explained in Al khatib et al. (2014) for the morphological examination. The vouchers are deposited in the following institutions and private collections: AICF, Lucian Fusu collection, Al. I. Cuza University, Iasi, Romania; BMNH, Natural History Museum, London, UK; CBGP, Centre for Biology and Management of Populations, Montpellier, France; CNC, Canadian National Collection of Insects, Arachnids and Nematodes, Agriculture \& Agri-food Canada, Ottawa, ON, Canada; FALPC, Fadel Al khatib personal collection, Faculty of Agricultural Engineering, University of Aleppo, Syria; GDPC, Gérard Delvare personal collection, Montpellier, France; MNHG, Museum of Natural History of Geneva, Switzerland; MNHN, National Museum of Natural History, Paris, France; NHRS, Naturhistoriska riksmuseet, Stockholm, Sweden. The depository's acronyms of voucher specimens are included in (Additional file 1: Table S2; Additional file Dryad: doi:10.5061/dryad.115m1). Sampling information (host-plants, collection dates, and localities) is listed in Table 1.

\section{Marker choice}

Seven markers displaying various rates of molecular evolution were used: two coding portions of mitochondrial genes (Cytochrome oxidase I, COI and Cytochrome b, $C y t b)$, two coding regions of nuclear genes (the F2 copy of elongation factor 1-alpha, EF-1 $\alpha$ and Wingless, $W g$ ) and three (at least partially) non-coding regions of other nuclear genes (the mitotic checkpoint control protein, Bub3; the ribosomal protein L27a, $R p L 27 a$, and the ribosomal protein S4, RpS4). All these markers were previously used for phylogenetic analyses in arthropods. COI and Cytb have been used to resolve insect molecular phylogenies at shallower taxonomic levels [38-41]. The $W g$ gene has provided a useful tool for the reconstruction of phylogenetic relationships at lower to intermediate taxonomic levels in different insect groups [32, 38, 41-45]. EF-1 $\alpha$ has proven to evolve at slow rates and provide phylogenetic information at deeper levels (i.e. family relationships) [39, 46-51]. The Bub3 gene is more rarely used $[52,53]$ for inferring phylogenetic relationships at a similar taxonomic level as $\mathrm{Wg}$. Finally, ribosomal proteins $R p L 27 a$ and $R p S 4$ have been used with success to infer the phylogeny of Hymenoptera associated with oak galls or figs $[39,54-56]$.

\section{DNA extraction, PCR amplification and sequencing}

Genomic DNA was extracted from a single individual using the Qiagen DNeasy kit (Hilden, Germany) with some minor modifications with regard to the manufacturer's protocol. Entire specimens were incubated at $56{ }^{\circ} \mathrm{C}$ for $15-17 \mathrm{~h}$ and DNA extraction was performed without destruction of the specimens, to allow subsequent examination of morphology (see $\mathbb{S}$ Sampling). Primer sequences are given in Additional file 1: Table S1.

For the two mitochondrial genes $(\mathrm{COI}$ and $\mathrm{Cytb})$, the PCR mix was prepared in $20 \mu \mathrm{l}$ as follows: $1 \mu \mathrm{l}$ of DNA $(1-55 \mathrm{ng} / \mu \mathrm{l}), 14.64 \mu \mathrm{l}$ of Milli-Q water, $2 \mu \mathrm{l}$ of $10 \mathrm{x}$ PCR buffer containing $\mathrm{MgCl} 2(1 \mathrm{x}), 1 \mu \mathrm{l}$ of $10 \mu \mathrm{M}$ primer cocktail $(0.5 \mu \mathrm{M}), 0.16 \mu \mathrm{l}$ of dNTPs $25 \mathrm{mM}$ each $(0.2 \mathrm{mM})$ and $0.2 \mu \mathrm{l}$ of $5 \mathrm{U} / \mu \mathrm{l}$ Taq DNA Polymerase (Qiagen, Hilden, Germany).

For the nuclear genes (Bub3, EF1- $\alpha, R p L 27 a, R p S 4$ and $W g$ ), the PCR mix was realised in $25 \mu \mathrm{l}$ as follows: $2 \mu \mathrm{l}$ of DNA (1-55 ng/ $\mu \mathrm{l}), 19.825 \mu \mathrm{l}$ of Milli-Q water, $2.5 \mu \mathrm{l}$ of 10x PCR buffer containing $\mathrm{MgCl} 2(1 \mathrm{x}), 0.175 \mu \mathrm{l}$ of $100 \mu \mathrm{M}$ primer cocktail $(0.7 \mu \mathrm{M}), 0.2 \mu \mathrm{l}$ of dNTPs $25 \mathrm{mM}$ each $(0.2 \mathrm{mM})$ and $0.125 \mu \mathrm{l}$ of $5 \mathrm{U} / \mu \mathrm{l}$ Taq DNA Polymerase (Qiagen, Hilden, Germany).

PCR conditions for $\mathrm{Wg}$ and $\mathrm{COI}$ were as described in [32]. Those for other genes were as follows: $\mathrm{Cytb}: 94{ }^{\circ} \mathrm{C}$ for $5 \mathrm{~min}$, followed by 40 cycles of (i) $94{ }^{\circ} \mathrm{C}$ for $1 \mathrm{~min}$, (ii) $50{ }^{\circ} \mathrm{C}$ for $1 \mathrm{~min}$, and (iii) $72{ }^{\circ} \mathrm{C}$ for $90 \mathrm{~s}$ with a final extension at $72{ }^{\circ} \mathrm{C}$ for $10 \mathrm{~min}$; nuclear markers: $94{ }^{\circ} \mathrm{C}$ for $4 \mathrm{~min}$, followed by 40 cycles of (i) $94{ }^{\circ} \mathrm{C}$ for $30 \mathrm{~s}$, (ii) $58{ }^{\circ} \mathrm{C}$ for $E F-1 \alpha, 48^{\circ} \mathrm{C}$ for Bub3, $57^{\circ} \mathrm{C}$ for $R p S 4$ and $55^{\circ} \mathrm{C}$ for $R p L 27 a$, (iii) $72{ }^{\circ} \mathrm{C}$ for $5 \mathrm{~min}$ with final extension at $72{ }^{\circ} \mathrm{C}$ for $5 \mathrm{~min}$.

In the absence of amplification or if the signal was too weak, we improved yields of PCRs by using 2x QIAGEN Multiplex PCR Master Mix (Qiagen, Hilden, Germany). In this case, PCRs were performed in a $25 \mu \mathrm{l}$ reaction volume: $2 \mu \mathrm{l}$ of DNA, $16.5 \mu \mathrm{l}$ of Milli-Q water, $0.125 \mu \mathrm{l}$ of $100 \mu \mathrm{M}$ primer cocktail $(0.5 \mu \mathrm{M})$ and $6.25 \mu \mathrm{l}$ of $2 \mathrm{x}$ QIAGEN Multiplex PCR Master Mix (1x) and PCR conditions were as specified in the QIAGEN $^{\circ}$ Multiplex PCR kit: $95{ }^{\circ} \mathrm{C}$ for $15 \mathrm{~min}$, followed by 40 cycles of (i) $95{ }^{\circ} \mathrm{C}$ for $30 \mathrm{~s}$, (ii) $48{ }^{\circ} \mathrm{C}-58{ }^{\circ} \mathrm{C}$ for $90 \mathrm{~s}$, (iii) $72{ }^{\circ} \mathrm{C}$ for $1 \mathrm{~min}$, with final extension at $72{ }^{\circ} \mathrm{C}$ for $10 \mathrm{~min}$.

All PCRs were performed on a GeneAmp 9700 thermocycler. PCR products were visualized using the QIAxcel Advanced System and QIAxcel DNA Fast Analysis Kit (Qiagen). PCR products were sent to GENOSCREEN (Lille, France) or to BECKMAN COULTER GENOMICS (Stansted, United Kingdom) for sequencing in both 
Table 1 Sample information for the specimens included in the phylogenetic analysis

\begin{tabular}{|c|c|c|c|c|c|c|c|c|c|c|}
\hline Species & $\begin{array}{l}\text { Collection } \\
\text { code }\end{array}$ & $\begin{array}{l}\text { Molecular } \\
\text { code }\end{array}$ & Country & Department & City & $\mathrm{N}^{\circ}$ & $E^{\circ}$ & Host insect & Associated plant & $\begin{array}{l}\text { Collection } \\
\text { date }\end{array}$ \\
\hline Eupelmus acinellus & FAL1363 & 10235 & France & Aude & Durban-Corbières & $42.99825^{\circ}$ & $2.80690^{\circ}$ & Mesophleps oxycedrella & Juniperus oxycedrus & March 2012 \\
\hline Eupelmus acinellus & FAL1366 & 10237 & France & Var & Fayence & $43.65513^{\circ}$ & $6.68813^{\circ}$ & Mesophleps oxycedrella & Juniperus oxycedrus & March 2012 \\
\hline Eupelmus annulatus & FAL1176 & 10198 & France & Alpes-Maritimes & $\begin{array}{l}\text { Gréolières-les- } \\
\text { Neiges }\end{array}$ & $43.81584^{\circ}$ & $6.88711^{\circ}$ & Diplolepis rosae & Rosa canina & March 2012 \\
\hline Eupelmus annulatus & NB783 & 10354 & France & Gard & Le Castanet & $43.98925^{\circ}$ & $3.70094^{\circ}$ & Dryocosmus kuriphilus & Castanea sativa & $\begin{array}{l}\text { February } \\
2012\end{array}$ \\
\hline Eupelmus annulatus & GDEL4053 & 10041 & Hungary & Veszprém & Hegyesd & $46.933333^{\circ}$ & $17.522778^{\circ}$ & Unknown & On Quercus cerris & June 2010 \\
\hline Eupelmus annulatus & LF.an.SW 01 & 10471 & Sweden & Öland & Mörbylånga & $56.61670^{\circ}$ & $16.507617^{\circ}$ & Unknown & Unknown & $\begin{array}{l}\text { August } \\
2006\end{array}$ \\
\hline Eupelmus azureus & FAL1323 & 10222 & France & Ardèche & $\begin{array}{l}\text { Saint-Georges-les- } \\
\text { Bains }\end{array}$ & $44.85028^{\circ}$ & $4.82433^{\circ}$ & Biorhiza pallida & Quercus pubescens & June 2012 \\
\hline Eupelmus azureus & NB773a & 10361 & France & Var & La Garde-Freinet & $43.30487^{\circ}$ & $6.43701^{\circ}$ & Dryocosmus kuriphilus & Castanea sativa & $\begin{array}{l}\text { February } \\
2012\end{array}$ \\
\hline Eupelmus azureus & GDEL4048 & 10034 & Italy & Piemonte/Cuneo & Palanfré & $44.165833^{\circ}$ & $7.50361^{\circ} 1$ & Unknown & Unknown & $\begin{array}{l}\text { August } \\
2010\end{array}$ \\
\hline Eupelmus azureus & L.Loru713 & 10245 & Italy & Sardinia & Aritzo & $39.94743^{\circ}$ & $9.19968^{\circ}$ & Dryocosmus kuriphilus & Castanea sativa & $\begin{array}{l}\text { August } \\
2011\end{array}$ \\
\hline Eupelmus azureus & PJ10077-21-4 & 10575 & Hungary & Vezprém & Várpalota & $47.19809^{\circ}$ & $18.21204^{\circ}$ & Andricus solitarius & $\begin{array}{l}\text { Quercus pubescens/ } \\
\text { Q. cerris }\end{array}$ & June 2010 \\
\hline Eupelmus azureus & PJ11054-2-2 & 10578 & Turkey & Bursa & Güneybudaklar & $40.00560^{\circ}$ & $29.14982^{\circ}$ & Andricus fecundator & Quercus sp. & - \\
\hline Eupelmus azureus & MC-C4 & 10486 & Switzerland & Stabio & Via Roccoletta & $45.84722^{\circ}$ & $8.92638^{\circ}$ & Dryocosmus kuriphilus & Castanea sativa & $\begin{array}{l}\text { August } \\
2012\end{array}$ \\
\hline Eupelmus cerris & GDEL4109 & 10118 & Hungary & Vezprém & Hegyesd & $46.93333^{\circ}$ & $17.52278^{\circ}$ & Unknown & On Quercus cerris & June 2010 \\
\hline Eupelmus confusus & FAL1278 & 10443 & France & Ardèche & $\begin{array}{l}\text { Saint-Georges- } \\
\text { Montpellier }\end{array}$ & $43.6104^{\circ}$ & $3.77227^{\circ}$ & Bactrocera oleae & Olea europaea & $\begin{array}{l}\text { October } \\
2011\end{array}$ \\
\hline Eupelmus confusus & FAL1519 & 10412 & France & Haute-Corse & Lumio & $42.55879^{\circ}$ & $8.81299^{\circ}$ & Bactrocera oleae & Olea europaea & $\begin{array}{l}\text { September } \\
2012\end{array}$ \\
\hline Eupelmus confusus & FAL1051 & 10145 & Italy & Liguria & Bussana-Vecchia & $43.84026^{\circ}$ & $7.82905^{\circ}$ & Myopites stylata & Dittrichia viscosa & $\begin{array}{l}\text { January } \\
2011\end{array}$ \\
\hline Eupelmus confusus & FAL1108 & 10250 & Spain & Logroño & La Rioja & - & - & Myopites stylata & Dittrichia viscosa & March 2012 \\
\hline Eupelmus confusus & LF.ma.GR 01 & 10425 & Greece & Seres & $\begin{array}{l}\text { Kerkini Lake } \\
\text { Nat.Park }\end{array}$ & $41.27833^{\circ}$ & $23.21955^{\circ}$ & Unknown & Unknown & June 2008 \\
\hline Eupelmus confusus & LF.ma.GR 02 & 10426 & Greece & Seres & Kerkini lake & $41.20180^{\circ}$ & $23.07747^{\circ}$ & Unknown & Unknown & $\begin{array}{l}\text { September } \\
2007\end{array}$ \\
\hline Eupelmus confusus & GDEL4173 & 10596 & France & Hérault & Laroque & $45.91722^{\circ}$ & $3.74361^{\circ}$ & Unknown & $\begin{array}{l}\text { On Quercus } \\
\text { pubescens }\end{array}$ & July 2013 \\
\hline Eupelmus confusus & LF.ma.IR 05 & 10424 & Iran & Kerman & Bidkhan & $29.59725^{\circ}$ & $56.48600^{\circ}$ & Unknown & On Salix alba & May 2012 \\
\hline
\end{tabular}


Table 1 Sample information for the specimens included in the phylogenetic analysis (Continued)

\begin{tabular}{|c|c|c|c|c|c|c|c|c|c|c|c|}
\hline Eupelmus & confusus & LF.ma.CY 01 & 10427 & Cyprus & Lemesos & Lemesos & $34.73189^{\circ}$ & $33.05175^{\circ}$ & $\begin{array}{l}\text { Apomyelois ceratoniae \& } \\
\text { Asphondylia gennadii }\end{array}$ & Ceratonia siliqua & May 2009 \\
\hline Eupelmus & fulvipes & FAL1221 & 10200 & France & Alpes-Maritimes & $\begin{array}{l}\text { Gréolières-les- } \\
\text { Neiges }\end{array}$ & $43.81584^{\circ}$ & $6.88711^{\circ}$ & Diplolepis rosae & Rosa canina & March 2012 \\
\hline Eupelmus & fulvipes & LF.ro.RO 02 & 10656 & Romania & Constanța & $\begin{array}{l}\text { Hagieni \& Negru } \\
\text { Voda }\end{array}$ & - & - & Diplolepis spinosissimae & Rosa sp. & - \\
\hline Eupelmus & fulvipes & LF.ro.GE 01 & 10657 & Germany & $\begin{array}{l}\text { Rottenburg- } \\
\text { Wurmlingen }\end{array}$ & & - & - & Diplolepis rosae & Rosa sp. & $\begin{array}{l}\text { October } \\
2011\end{array}$ \\
\hline Eupelmus & gemellus & FAL1260 & 10438 & France & Var & Porquerolles & $42.99534^{\circ}$ & $6.2044^{\circ}$ & Bactrocera oleae & Olea europaea & - \\
\hline Eupelmus & gemellus & FAL1359 & 10230 & France & Alpes-Maritimes & Biot & $43.63455^{\circ}$ & $7.082490^{\circ}$ & Mesophleps oxycedrella & Juniperus oxycedrus & March 2012 \\
\hline Eupelmus & gemellus & NB441 & 10415 & France & Haute-Corse & Bisinchi & $42.48983^{\circ}$ & $9.32797^{\circ}$ & Dryocosmus kuriphilus & Castanea sativa & June 2012 \\
\hline Eupelmus & gemellus & FAL1004 & 10130 & Italy & Liguria & Bussana-Vecchia & $43.84026^{\circ}$ & $7.82905^{\circ}$ & Myopites stylata & Dittrichia viscosa & $\begin{array}{l}\text { January } \\
2011\end{array}$ \\
\hline Eupelmus & gemellus & FAL1508 & 10405 & Italy & Sardinia & $\begin{array}{l}\text { Province } \\
\text { d'Oristano }\end{array}$ & $39.70041^{\circ}$ & $8.739690^{\circ}$ & Unknown & On Pistacia lentiscus & $\begin{array}{l}\text { October } \\
2012\end{array}$ \\
\hline Eupelmus & janstai & GDEL4046 & 10032 & $\begin{array}{l}\text { Czech } \\
\text { Republic }\end{array}$ & Břeclav & Pavlov & $48.867500^{\circ}$ & $16.654166^{\circ}$ & Unknown & On T. platyphyllos & July 2010 \\
\hline Eupelmus & kiefferi & NB674b & 10341 & France & Alpes-Maritimes & Granile & $44.03942^{\circ}$ & $7.57575^{\circ}$ & Dryocosmus kuriphilus & Castanea sativa & March 2012 \\
\hline Eupelmus & kiefferi & NB666 & 10325 & France & Haute-Corse & Muratu & $42.55139^{\circ}$ & $9.30929^{\circ}$ & Dryocosmus kuriphilus & Castanea sativa & $\begin{array}{l}\text { December } \\
2012\end{array}$ \\
\hline Eupelmus & kiefferi & FAL1070 & 10151 & Italy & Liguria & Bussana-Vecchia & $43.84026^{\circ}$ & $7.82905^{\circ}$ & Myopites stylata & Dittrichia viscosa & $\begin{array}{l}\text { January } \\
2012\end{array}$ \\
\hline Eupelmus & kiefferi & FAL1109 & 10167 & Spain & Logroño & La Rioja & - & - & Myopites stylata & Dittrichia viscosa & March 2012 \\
\hline Eupelmus & kiefferi & FAL1511 & 10406 & Lebanon & Bakhoun & Fanar & - & - & Myopites stylata & Dittrichia viscosa & March 2012 \\
\hline Eupelmus & kiefferi & GDEL4045 & 10030 & Hungary & Szombathely & Köszeg & $47.363888^{\circ}$ & $16.52500^{\circ}$ & Unknown & On Salix cinerea & June 2010 \\
\hline Eupelmus & kiefferi & MC-C124 & 10492 & Switzerland & Riviera & Monte Ceneri & $46.136944^{\circ}$ & $08.902500^{\circ}$ & Dryocosmus kuriphilus & Castanea sativa & July 2012 \\
\hline Eupelmus & kiefferi & LF.ma.RO 01 & 10423 & Romania & Botoşani & Leorda & - & - & Unknown & Unknown & July 2007 \\
\hline Eupelmus & kiefferi & ZL.fu.RO 05 & 10585 & Romania & Mures & Sovata & $46.54482^{\circ}$ & $24.96769^{\circ}$ & Diplolepis mayri & Rosa canina & March 2012 \\
\hline Eupelmus & kiefferi & LF.fu.GE 02 & 10658 & Germany & $\begin{array}{l}\text { Rottenburg- } \\
\text { Wurmlingen }\end{array}$ & & - & - & Diplolepis rosae & Rosa sp. & $\begin{array}{l}\text { October } \\
2013\end{array}$ \\
\hline Eupelmus & kiefferi & LF.fu.SL 01 & 10467 & Slovakia & Muranska Planina & Predna Hora & - & - & Unknown & Unknown & July 2009 \\
\hline Eupelmus & kiefferi & GDEL4043 & 10028 & $\begin{array}{l}\text { Czech } \\
\text { Republic }\end{array}$ & Trutnov & Vilantice & $50.365833^{\circ}$ & $15.737222^{\circ}$ & Unknown & Unknown & July 2010 \\
\hline Eupelmus & kiefferi & LF.fu.ES 01 & 10463 & Estonia & Tartu & Rannu Parish & - & - & Unknown & Unknown & June 2010 \\
\hline Eupelmus & kiefferi & FAL1524 & 10593 & Algeria & Tigzirt & Tigzirt & - & - & Myopites stylata & Dittrichia viscosa & $\begin{array}{l}\text { Februry } \\
2013\end{array}$ \\
\hline Eupelmus & longicalvus & GDEL4038 & 10019 & Italy & $\begin{array}{l}\text { Friuli Venezia } \\
\text { Giulia }\end{array}$ & Chiusaforte & $46.405277^{\circ}$ & $13.445000^{\circ}$ & Unknown & Unknown & July 2008 \\
\hline
\end{tabular}


Table 1 Sample information for the specimens included in the phylogenetic analysis (Continued)

\begin{tabular}{|c|c|c|c|c|c|c|c|c|c|c|c|}
\hline Eupelmus & longicalvus & LF.ma.SW 02 & 10429 & Sweden & Gotland & $\begin{array}{l}\text { Gotlands } \\
\text { commun }\end{array}$ & $57^{\circ} 32.207^{\prime}$ & $18^{\circ} 20.273^{\prime}$ & Unknown & Unknown & July 2004 \\
\hline Eupelmus & longicalvus & GDEL4191 & 10603 & Italy & $\begin{array}{l}\text { Friuli-Venezia } \\
\text { Giulia }\end{array}$ & Chiusaforte & $46.39944^{\circ}$ & $13.45944^{\circ}$ & Unknown & Unknown & July 2008 \\
\hline Eupelmus & minozonus & GDEL4030 & 10009 & Hungary & Veszprém & Hegyesd & $46.93333^{\circ}$ & $17.52278^{\circ}$ & Unknown & On Quercus cerris & June 2010 \\
\hline Eupelmus & minozonus & GDEL4030 & 10010 & Hungary & Veszprém & Hegyesd & $46.93333^{\circ}$ & $17.52278^{\circ}$ & Unknown & On Quercus cerris & June 2010 \\
\hline Eupelmus & minozonus & GDEL4030 & 10011 & Hungary & Veszprém & Hegyesd & $46.93333^{\circ}$ & $17.52278^{\circ}$ & Unknown & On Quercus cerris & June 2010 \\
\hline Eupelmus & opacus & LF.ur.GR 01 & 10459 & Greece & Seres & Krousia Mts site & $41^{\circ} 11^{\prime} 32,4^{\prime \prime}$ & $23^{\circ} 03^{\prime} 59,5^{\prime \prime}$ & Unknown & Unknown & June 2007 \\
\hline Eupelmus & opacus & LF.ur.SW 02 & 10460 & Sweden & Östergötland & $\begin{array}{l}\text { Ödeshögs } \\
\text { kommun }\end{array}$ & $58^{\circ} 18.452^{\prime}$ & $14^{\circ} 37.859^{\prime}$ & Unknown & Unknown & $\begin{array}{l}\text { August } \\
2005\end{array}$ \\
\hline Eupelmus & pistaciae & GDEL4027 & 10004 & France & Hérault & Cazevieille & $43.752222^{\circ}$ & $3.770000^{\circ}$ & Megastigmus pistaciae & Pistacia terebinthus & $\begin{array}{l}\text { October } \\
2010\end{array}$ \\
\hline Eupelmus & pistaciae & GDEL4027 & 10005 & France & Hérault & Cazevieille & $43.752222^{\circ}$ & $3.770000^{\circ}$ & Megastigmus pistaciae & Pistacia terebinthus & $\begin{array}{l}\text { October } \\
2010\end{array}$ \\
\hline Eupelmus & pistaciae & GDEL4027 & 10507 & France & Hérault & Cazevieille & $43.752222^{\circ}$ & $3.770000^{\circ}$ & Megastigmus pistaciae & Pistacia terebinthus & $\begin{array}{l}\text { October } \\
2010\end{array}$ \\
\hline Eupelmus & priotoni & GDEL4051 & 10038 & France & Aveyron & Sauclières & $43.96389^{\circ}$ & $3.355833^{\circ}$ & Unknown & Unknown & June 2011 \\
\hline Eupelmus & purpuricollis & LF.ur.GR 02 & 10650 & Greece & Seres & nr Neo Petritsi & $41^{\circ} 18^{\prime} 49,8^{\prime \prime}$ & $23^{\circ} 16^{\prime} 35,6^{\prime \prime}$ & Unknown & Unknown & July 2008 \\
\hline Eupelmus & purpuricollis & LF.ur.GR 03 & 10651 & Greece & Seres & Kerkini & $41^{\circ} 11^{\prime} 32,4^{\prime \prime}$ & $23^{\circ} 03^{\prime} 59,5^{\prime \prime}$ & Unknown & Unknown & July 2007 \\
\hline Eupelmus & simizonus & GDEL4142 & 10297 & France & Ardèche & Les Vans & $44.387222^{\circ}$ & $4.154444^{\circ}$ & Unknown & $\begin{array}{l}\text { On Quercus } \\
\text { pubescens }\end{array}$ & July 2012 \\
\hline Eupelmus & tibicinis & GDEL4148 & 10299 & France & Ardèche & Chassagnes & $44.403888^{\circ}$ & $4.178333^{\circ}$ & Unknown & $\begin{array}{l}\text { On Quercus } \\
\text { pubescens }\end{array}$ & July 2012 \\
\hline Eupelmus & tibicinis & GDEL4149 & 10300 & France & Ardèche & $\begin{array}{l}\text { Berrias-et- } \\
\text { Casteljau }\end{array}$ & $44.39389^{\circ}$ & $4.194722^{\circ}$ & Unknown & Unknown & July 2012 \\
\hline Eupelmus & tibicinis & GDEL4175 & 10598 & France & Hérault & Laroque & $45.91722^{\circ}$ & $3.74361^{\circ}$ & Unknown & $\begin{array}{l}\text { On Quercus } \\
\text { pubescens }\end{array}$ & July 2013 \\
\hline Eupelmus & urozonus & NB677 & 10333 & France & Lot & Aynac & $44.78155^{\circ}$ & $1.85896^{\circ}$ & Dryocosmus kuriphilus & Castanea sativa & $\begin{array}{l}\text { January } \\
2012\end{array}$ \\
\hline Eupelmus & urozonus & FAL1518 & 10410 & France & Haute-Corse & Lumio & $42.55879^{\circ}$ & $8.81299^{\circ}$ & Bactrocera oleae & Olea europaea & $\begin{array}{l}\text { September } \\
2012\end{array}$ \\
\hline Eupelmus & urozonus & FAL1060 & 10148 & Italy & Liguria & Bussana-Vecchia & $43.84026^{\circ}$ & $7.82905^{\circ}$ & Myopites stylata & Dittrichia viscosa & $\begin{array}{l}\text { January } \\
2011\end{array}$ \\
\hline Eupelmus & urozonus & L.Loru235 & 10241 & Italy & Sardinia & Desulo & $39.99198^{\circ}$ & $9.23053^{\circ}$ & Dryocosmus kuriphilus & Castanea sativa & July 2011 \\
\hline Eupelmus & urozonus & FAL1106 & 10165 & Spain & Logroño & La Rioja & - & - & Myopites stylata & Dittrichia viscosa & March 2012 \\
\hline Eupelmus & urozonus & NB1117 & 10251 & Greece & Crete & Gournes & $35.32822^{\circ}$ & $25.28388^{\circ}$ & Myopites stylata & Dittrichia viscosa & March 2012 \\
\hline Eupelmus & urozonus & MC-C100 & 10488 & Switzerland & Riviera & Monte Ceneri & $46.136944^{\circ}$ & $8.902500^{\circ}$ & Dryocosmus kuriphilus & Castanea sativa & July 2012 \\
\hline
\end{tabular}


Table 1 Sample information for the specimens included in the phylogenetic analysis (Continued)

\begin{tabular}{|c|c|c|c|c|c|c|c|c|c|c|}
\hline Eupelmus urozonus & PJ10077-2-6 & 10573 & Hungary & Vezprém & Várpalota & $47.198091^{\circ}$ & $18.21204^{\circ}$ & Andricus lucidus & $\begin{array}{l}\text { Quercus pubescens/ } \\
\text { Q. cerris }\end{array}$ & $\begin{array}{l}\text { November } \\
2010\end{array}$ \\
\hline Eupelmus urozonus & LF.fu.RO 01 & 10464 & Romania & Neamts & Podoleni & & & Unknown & Unknown & $\begin{array}{l}\text { September } \\
2012\end{array}$ \\
\hline Eupelmus urozonus & LF.ur.IR 02 & 10457 & Iran & Kerman & Bidkhan & - & - & Unknown & Ephedra sp. & March 2010 \\
\hline Eupelmus vindex & GDEL4054 & 10042 & Hungary & Veszprém & Hegyesd & - & - & Unknown & Unknown & June 2010 \\
\hline Eupelmus vindex & LF.vi.RO 02 & 10468 & Romania & laşi & Iaşi & - & - & Unknown & Unknown & June 2007 \\
\hline Eupelmus vindex & LF.vi.RO 01 & 10469 & Romania & Tulcea & Letea & - & - & Unknown & Unknown & May 2007 \\
\hline Eupelmus microzonus & GDEL4116 & 10192 & France & Haute-Corse & Aléria & $42.128611^{\circ}$ & $9.465556^{\circ}$ & Bruchophagus sp. & Asphodelus ramosus & $\begin{array}{l}\text { September } \\
2011\end{array}$ \\
\hline $\begin{array}{l}\text { Eupelmus } \\
\text { atropurpureus }\end{array}$ & PJ11159_23_1 & 10580 & Spain & Aragón & Huesca & & & Unknown & Poaceae & $\begin{array}{l}\text { November } \\
2011\end{array}$ \\
\hline Eupelmus pini & GDEL4058 & 10048 & France & Alpes-Maritimes & Guillaumes & $44.070833^{\circ}$ & $6.853056^{\circ}$ & Unknown & $\begin{array}{l}\text { Dead trunk of Pinus } \\
\text { sylvestris }\end{array}$ & $\begin{array}{l}\text { August } \\
2009\end{array}$ \\
\hline Eupelmus matranus & FAL1491 & 10318 & France & Alpes-Maritimes & Sophia-Antipolis & $43.61671^{\circ}$ & $7.07550^{\circ}$ & Unknown & On Quercus ilex & $\begin{array}{l}\text { October } \\
2012\end{array}$ \\
\hline Eupelmus falcatus & GDEL4088 & 10090 & Hungary & Veszprém & Nagavászony & $47.021667^{\circ}$ & $17.724167^{\circ}$ & Unknown & Unknown & June 2010 \\
\hline Eupelmus seculatus & GDEL4089 & 10091 & France & Gard & Beauvoisin & $43.712500^{\circ}$ & $4.307222^{\circ}$ & Unknown & Unknown & $\begin{array}{l}\text { August } \\
2011\end{array}$ \\
\hline Eupelmus linearis & GDEL4069 & 10062 & France & Lozère & Cocurès & $45.30555^{\circ}$ & $4.59194^{\circ}$ & Unknown & Unknown & July 2011 \\
\hline Eupelmus linearis & GDEL4073 & 10066 & Hungary & Veszprém & Nagavászony & $47.021667^{\circ}$ & $17.724167^{\circ}$ & Unknown & Unknown & June 2010 \\
\hline $\begin{array}{l}\text { Eupelmus } \\
\text { testaceiventris }\end{array}$ & GDEL4078 & 10075 & Cameroon & Adamaoua & Osséré Gadou & $7.173056^{\circ}$ & $13.623056^{\circ}$ & Unknown & Unknown & $\begin{array}{l}\text { November } \\
2008\end{array}$ \\
\hline $\begin{array}{l}\text { Eupelmus juniperinus } \\
\text { thuriferae }\end{array}$ & GDEL4064 & 10057 & France & Hautes-Alpes & Saint-Crépin & $44.710556^{\circ}$ & $6.606389^{\circ}$ & Unknown & $\begin{array}{l}\text { On Juniperus } \\
\text { thurifera }\end{array}$ & $\begin{array}{l}\text { August } \\
2008\end{array}$ \\
\hline Reikosiella aff. rostrata & NB670 & 10336 & France & Drôme & Génissieux & $45.09059^{\circ}$ & $5.07161^{\circ}$ & Dryocosmus kuriphilus & Castanea sativa & $\begin{array}{l}\text { February } \\
2012\end{array}$ \\
\hline Reikosiella aff. rostrata & NB810 & 10350 & France & Alpes-Maritimes & Tende & $44.056689^{\circ}$ & $7.579353^{\circ}$ & Dryocosmus kuriphilus & Castanea sativa & March 2012 \\
\hline Anastatus sidereus & GDEL4098 & 10105 & France & Alpes-Maritimes & Fontan & $44.026389^{\circ}$ & $7.577778^{\circ}$ & Unknown & Unknown & July 2010 \\
\hline $\begin{array}{l}\text { Anastatus aff. } \\
\text { temporalis }\end{array}$ & GDEL4100 & 10107 & France & Gard & Générac & $43.719444^{\circ}$ & $4.353611^{\circ}$ & Unknown & Unknown & $\begin{array}{l}\text { August } \\
2011\end{array}$ \\
\hline
\end{tabular}


directions. All sequences were deposited in GenBank (Additional file 1: Table S2).

\section{Sequence alignment and phylogenetic analysis Alignment}

Sequences were aligned using Muscle [57] with the default settings as implemented in SeaView v4.4.1 [58] and subsequently visually checked. To assess the impact of indels on the phylogenetic resolution, highly divergent blocks present in Bub3, RpS4 and RpL27a alignments were either included in or excluded from the analyses. These blocks were removed using Gblocks [59] with the default settings as implemented in SeaView. Alignments of COI, Cytb, EF-1 $\alpha$ and $\mathrm{Wg}$ were translated to amino acids using Mega v5.1 [60] to detect potential frameshift mutations and premature stop codons, which may indicate the presence of pseudogenes.

\section{Gene by gene analysis}

To detect (i) possible inconsistencies linked to contamination during laboratory procedures, (ii) poor-quality sequences, (iii) possible pseudogenes or other artefacts, and (iv) to evaluate the impact of the Gblock procedure on the individual phylogenetic resolution, genes were first analysed separately using a maximum likelihood approach (ML).

\section{Concatenated datasets analysis}

Phylogenetic analyses were performed on concatenated nucleotide sequences using both $\mathrm{ML}$ and Bayesian methods. Four partitioning schemes were compared: (i) two partitions: one for the two mitochondrial genes $(C O I$ and $C y t b)$ and another for all nuclear markers ( $W g, E F-1 \alpha, B u b 3, R p S 4$ \& RpL27a); (ii) six partitions: one for the two mitochondrial markers (COI and Cytb) and one for each nuclear marker ( $W g, E F-1 \alpha, B u b 3, R p S 4$ and $R p L 27 a$ ); (iii) seven partitions: one for the 1st and 2nd codon positions of the mtDNA, one for the 3rd codon positions of mtDNA, and one for each nuclear gene ( $W g, E F-1 \alpha, B u b, R p S 4$ and $R p L 27 a$ ); (iv) nine partitions: same as above with $W g$ and $E F-1 \alpha$ further partitioned by codon position (1st and 2nd codon positions versus 3rd positions).

Bayes factors (BF) $[61,62]$ were used to compare the four partitioning schemes. Harmonic means of the likelihood scores were used as estimators of the marginal likelihoods. Following [61] and [63], Bayes factors were calculated using the following formula: $\mathrm{BF}=2 \times(\operatorname{lnM} 1$ $\ln \mathrm{M} 0)+(\mathrm{P} 1-\mathrm{P} 0) \times \ln (0.01)$ where $\ln \mathrm{Mi}$ and $\mathrm{Pi}$ are the harmonic-mean of the ln likelihoods and the number of free parameters of the model $i$, respectively. BF values were interpreted following [61] and [62], with BF values between 2 and 6 , between 6 and 10 and higher than 10 indicating positive evidence, strong evidence, and very strong evidence favouring one model over the others respectively.

\section{Evolution models and phylogenetic reconstruction}

For the separated and concatenated datasets, the bestfitting model was identified using the Akaike information criterion (AIC) as implemented in jModelTest v0.1.1 [64].

For both gene-by-gene and concatenated analyses, maximum likelihood analyses and associated bootstrapping were performed using RAxML v8.0.9 [65]. The GTRCAT approximation of models was used for ML bootstrapping (1000 replicates). Bootstrap percentages (BP) $\geq 85 \%$ were considered as strong support and $\mathrm{BP}<65 \%$ as weak.

Bayesian analyses were performed only on the concatenated dataset using a parallel version of MrBayes v3.2.2 [66]. Model parameters for each data partition were independently estimated by unlinking parameters across partitions. Parameter values for the model were initiated with default uniform priors, and branch lengths were approximated using default exponential priors. Bayesian inferences were estimated using two simultaneous, independent runs of Markov Chain Monte Carlo (MCMC), including three heated and one cold chains. The Metropolis-coupled MCMC algorithm [67] was used to improve the mixing of Markov chains. Analyses were run for $20 \times 10^{6}$ generations with parameter values sampled every 2000 generations. To ensure convergence, $40 \times 10^{6}$ generations were used for the most complex partitioning scheme (9 partitions) with parameter values sampled every 4000 generations. To increase and improve the swap frequencies of states between cold and heated chains, the heating temperature (T) was set to 0.01 for the most complex partitioning scheme cleaned with Gblocks and to 0.02 for all other datasets. Convergence was assessed using the standard deviation of split frequencies given by MrBayes and the Effective Sample Size (ESS), as estimated using Tracer v1.6.0 [68]. The first $25 \%$ of the tree samples from the cold chain were discarded and considered as burn-in. Posterior probabilities $(\mathrm{PP}) \geq 0.95$ were considered as strong support and $\mathrm{PP}<0.90$ as weak.

Analyses were conducted using the CIPRES Science Gateway (www.phylo.org) [69].

\section{Evolutionary properties of marker sequences}

For each partition of the concatenated datasets (without Gblocks cleaning), base composition, substitution rates, and among sites rate variation $(\alpha)$ were estimated and compared. We also compared rate variation among partitions, considering the parameter $\mathrm{m}$ (rate multiplier).

\section{Comparative analysis \\ Evolution of ovipositor length}

The ovipositor of Hymenoptera is a complex organ that exhibits great interspecific variation (see for instance 
[23]). In species of Eupelmus, part of the ovipositor is easily visible at the extremity of the abdomen (the ovipositor sheaths), while the rest is concealed in the abdomen. The use of this visible part as a "proxy" of the total ovipositor length is a priori tempting in order to avoid damaging of specimens of newly described species known from very few individuals [32, 33]. In order to validate the use of this proxy, a total of 34 individuals of comparatively common species (e.g. E. azureus, E. confusus, E. gemellus, E. kiefferi, E. pistaciae, and E. urozonus) were dissected and, for each individual, we measured the length of the ovipositor stylet, the visible part of the ovipositor sheath and the metatibia (see dataset on Dryad: doi:10.5061/dryad.115m1). Measurements of the length of the ovipositor sheaths and hind tibia followed $\mathrm{Al}$ khatib et al. [32] (Additional file 2: Figure S18 A and C). The length of the ovipositor stylet (first and second valvulae) was measured from the articulation of the second valvula with the articulating bulb to the apex of the second valvula (Additional file 2: Figure S18 B). Using this dataset, we found evidence of linear relationships between the ovipositor sheath (response variable) and either the ovipositor stylet or the metatibia as predictors (data not shown). Moreover, no interaction was found between these two predictors and the host species (respectively $\mathrm{F}_{5 \mathrm{df}, 20 \mathrm{df}}=1.23$ with $p=0.34$ and $\mathrm{F}_{5 \mathrm{df}, 22 \mathrm{df}}=1.20$ with $p=0.34$ ). This suggests that the visible part of the ovipositor sheath can indeed be used as a reliable proxy of the entire ovipositor.

As a consequence, a first analysis was performed on the 19 species of the "E. urozonus species group" for which information about the ovipositor sheaths and the metatibia were available. This analysis includes a total of 121 individuals, with at least 2 individuals/species except for E. priotoni and E. simizonus (only one individual in each case). In most of the cases, we tried to select individuals from at least two geographical locations and/or, for generalist species, two host species (see dataset on Dryad: doi:10.5061/dryad.115m1). Both the absolute length of the ovipositor sheath ("AOS") and the ratio ("ROS") between the ovipositor sheaths and the metatibia were taken into account, the second one being potentially less sensitive to environmental-induced phenotypic plasticity (host and/or abiotic conditions). AOS/ ROS medians were then calculated for each Eupelmus species and these medians were used for the subsequent analysis (see below).

Two tests were then performed: (i) a Mantel test of the correlation between pairwise genetic distances ("phylogenetic matrix") and pairwise differences in AOS/ROS ("morphological matrix"). (Dis) similarities were estimated as $\left|d_{i}-d_{j}\right| /\left[\left(d_{i}+d_{j}\right) / 2\right]\left(d_{i}\right.$ and $d_{j}$ being the AOS/ROS medians obtained for species $i$ and $j$ respectively); (ii) the detection of a phylogenetic signal based on categories of AOS/ROS. For this purpose, "long ovipositors" (AOS/ROS exceeding the third quartile) were distinguished from "short ovipositors" (AOS/ ROS below this threshold). Briefly, the sum of state changes was calculated, leading to a D statistic that could be tested against two theoretical distributions: a phylogenetic randomness and a Brownian distribution, this latter being underlain by a continuous trait evolving along the phylogeny at a constant rate [70].

\section{Influence of phylogeny and ovipositor length on host range}

A second analysis was restricted to a subset of 13 species for which host range was also available. Most of the information about host range was obtained from $\mathrm{Al}$ khatib et al. [32] and from Gibson and Fusu (in prep). Jean Lecomte (comm. pers.) communicated the rearing of $E$. confusus from curculionid larvae. Taken as a whole, our host survey is probably not exhaustive but nevertheless encompassed a total of several thousands of individuals of the "E. urozonus species group" and, with regard to the host's diversity, 95 insect species representing 22 families and 6 orders (see dataset on Dryad: doi:10.5061/ dryad. $115 \mathrm{~m} 1$ ). Taken as a whole, these host insects were distributed on 18 plant families. Dissimilarities in host range were calculated-at three taxonomic levels (species, family and order) for the host insect and at one level (family) for the host plant-using the Bray-Curtis distance, each host taxon being treated qualitatively (at least one record versus none). This information was summarized and presented as "ecological matrices". Correlations between "phylogenetic", "morphological" and "ecological" matrices were tested using simple ( 2 matrices) or partial (3 matrices) Mantel tests, the relevance of these last tests having been repeatedly discussed (see for instance [71] and [72]).

Moreover, three kinds of traits were investigated using D-statistics (see previous paragraph):

(a) Host specificity ("specialists" which were reared from a single host species versus "generalists" that were reared from more than one host species). This specificity was evaluated at the order-family taxonomic level and at the species level. Because one may argue that our sampling underestimates specialists, we also performed this analysis under the assumption that all the rare species (E. janstai, E. longicalvus, E. minozonus, E. priotoni, E. purpuricollis, E. vindex) could be specialists.

(b)Ability ("Yes" or "No") to successfully parasitize some well-represented insect taxa at the ordinal level (Coleoptera, Diptera, Hymenoptera and Lepidoptera) or at the family level (Cynipidae within Hymenoptera and Cecidomyiidae within Diptera).

(c) The ability ("Yes" or "No") to exploit some main host plants (whatever the host insect), host plant being 


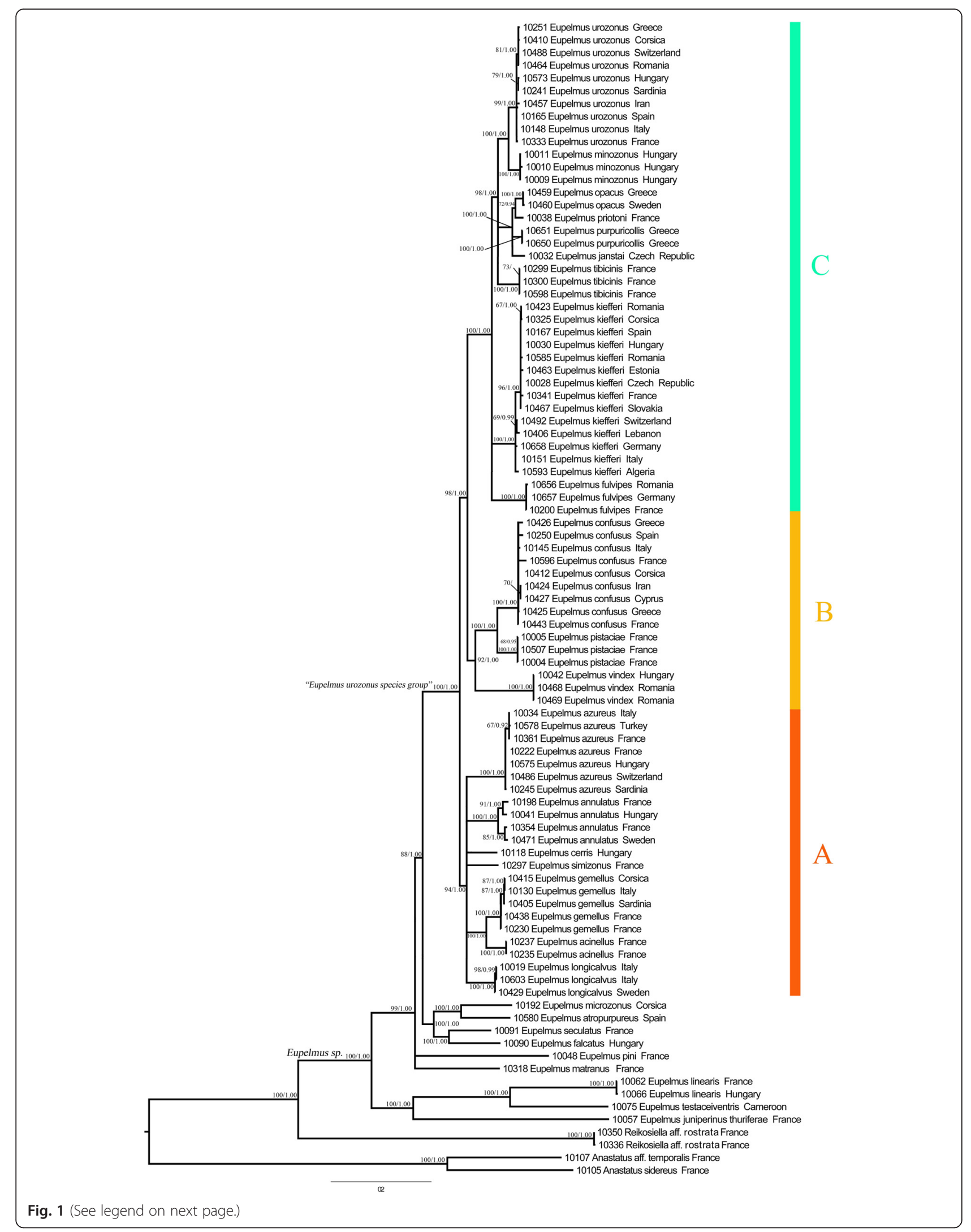


(See figure on previous page.)

Fig. 1 Phylogram of relationships among species of the "Eupelmus urozonus species group" obtained from the concatenated dataset alignment (5000 bp and 9 partitions) without the Gblocks cleaning of divergent blocks. Uppercase letters refer to clades discussed in the text. Nodes with likelihood bootstrap (BP) values <65 have been collapsed. BP $(\geq 65)$ and Bayesian posterior probabilities $(\geq 0.90)$ are indicated at nodes. Each line represents a sequenced individual with information in the following order: molecular code, species and country

treated at the family level (Asteraceae, Fagaceae, Rosaceae, Salicaceae, etc.).

\section{Software and packages}

Manipulations of files and statistical tests were conducted using the software R (http://www.R-project.org - version 3.0.3 - 2014-03-06) with the following packages "ade4" (Euclidian transformation of matrices) [73], "ape" (phylogeny) [74], "caper" (comparative analysis), "ecodist" (Mantel tests) [75] and "vegan" (similarities between host ranges) [76].

\section{Results}

\section{Alignments and single-marker analyses}

Successful amplification and sequencing was completed for all gene regions used in this study. However, sequencing failures occurred for some markers for a few individuals. Genbank accessions of the sequences obtained for all analysed genes are given in Additional file 1: Table S2. The final matrix contained 91 specimens. No stop codons, frame shifts, insertions or deletions were observed in coding gene regions.

The numbers of aligned base pairs, variable sites and parsimony-informative sites for each gene are summarized in Table 2. As expected, mitochondrial genes showed more parsimony-informative sites compared to nuclear markers (472 out of $1085 \mathrm{bp}$ ). Among the nuclear markers, $E F-1 \alpha$ exhibited the lowest number of variable and parsimony-informative sites (respectively 116 and 106 out of $517 \mathrm{bp}$ ). For RpL27a, removing the highly divergent alignment blocks significantly reduced the number of variable and parsimony-informative sites (from 54 to $38 \%$ for variable sites and from 34 to $30 \%$ for parsimony-informative sites). This loss consequently affected the resolution of the corresponding inferred topology (Additional file 2: Figure S16 and Figure S17). In contrast, the Gblocks procedure did not affect the number of variable and parsimonyinformative sites for $B u b 3$ and RpS4 and the resolution of the corresponding topologies (Additional file 2: Figures S12 - S15).

\section{Evolution models and partitions in the concatenated dataset}

Alignment lengths of the concatenated datasets with or without the exclusion of highly divergent blocks were
$3197 \mathrm{bp}$ and 5000 bp respectively. For all partitions, the best-fitting substitution model was the general time reversible model (GTR) with among-sites rate variation (ASRV) modelled by a discrete gamma distribution $(\Gamma)$ [77] for which we used four categories. For all Bayesian analyses, after discarding $25 \%$ of the samples as burn-in, the ESS value of each parameter largely exceeded 200, which indicated that convergence of runs was reached. Sixteen combined trees were obtained (Additional file 2: Figures S1 - S8). For all combined datasets, Bayes factors showed that the most complex partitioning scenario (9 partitions) was preferred over the three less complex ones (Table 3).

\section{Evolutionary properties of the markers}

Model parameter estimates for each partition of the Bayesian analysis of the "9 partitions without Gblocks cleaning dataset" are depicted in Table 4.

As expected, the mitochondrial partitions showed high base compositional bias ( 71.4 and $89.8 \%$ of $\mathrm{A} / \mathrm{T}$ for the first two positions and the third codon position respectively). Among the nuclear gene partitions, RpL27a, Bub3 and RpS4 were A/T-biased (77.9, 70 and $68.8 \%$ ) while

Table 2 Numbers and percentage of aligned base pairs, variable sites and parsimony-informative sites for the genes used in this study

\begin{tabular}{llll}
\hline Gene region & $\begin{array}{l}\text { Total } \\
\text { sites }\end{array}$ & sites & $\begin{array}{l}\text { Parsimony- } \\
\text { informative sites }\end{array}$ \\
\hline mtDNA & 1085 & $530(48.8 \%)$ & $472(43.5 \%)$ \\
Wg & 433 & $157(36.2 \%)$ & $147(33.9 \%)$ \\
EF-1a & 517 & $116(22.4 \%)$ & $106(20.5 \%)$ \\
$\begin{array}{l}\text { Bub3 alignment without } \\
\text { Gblocks }\end{array}$ & 481 & $161(33.4 \%)$ & $140(29.1 \%)$ \\
$\begin{array}{l}\text { Bub3 alignment with Gblocks } \\
\text { default }\end{array}$ & 391 & $132(33.7 \%)$ & $116(29.7 \%)$ \\
$\begin{array}{l}\text { RpS4 alignment without } \\
\text { Gblocks }\end{array}$ & 1259 & $451(35.8 \%)$ & $323(25.6 \%)$ \\
$\begin{array}{l}\text { RpS4 alignment with Gblocks } \\
\text { default }\end{array}$ & 525 & $189(36.0 \%)$ & $148(28.1 \%)$ \\
$\begin{array}{l}\text { RpL27a alignment without } \\
\text { Gblocks }\end{array}$ & 1225 & $661(53.9 \%)$ & $417(34.0 \%)$ \\
$\begin{array}{l}\text { RpL27a alignment with } \\
\text { Gblocks default }\end{array}$ & 246 & $93(37.8 \%)$ & $74(30.0 \%)$ \\
\hline
\end{tabular}


Table 3 Partitioning strategy selecting using Bayes factors (Harmonic-Mean) in Bayesian analyses

\begin{tabular}{|c|c|c|}
\hline Dataset partitioning models & Harmonic-mean (LnL) & Bayes factor \\
\hline \multicolumn{3}{|l|}{ Alignments without Gblocks } \\
\hline M1: mtDNA, nucDNA (2 partitions, 19 free parameters) & -38664.20 & $M 2, M 1=907.0$ \\
\hline M2: mtDNA, Wg, EF-1a, Bub3, RpS4, RpL27a (6 partitions, 59 parameters) & -38118.57 & $M 3, M 1=1909.5$ \\
\hline $\begin{array}{l}\text { M3: mtDNA 1\&2, mtDNA 3, Wg, EF-1a, Bub3, RpS4, RpL27a (7 partitions, } \\
69 \text { parameters) }\end{array}$ & -37594.33 & $M 3, M 2=1002.4$ \\
\hline \multirow{3}{*}{$\begin{array}{l}\text { M4: mtDNA 1\&2, mtDNA 3, Wg 1\&2, Wg 3, EF-1a 1\&2, EF-1a 3, Bub3, RpS4, } \\
\text { RpL27a (9 partitions, } 89 \text { parameters) }\end{array}$} & -37261.28 & $M 4, M 1=2483.5$ \\
\hline & & $M 4, M 2=1576.43$ \\
\hline & & $M 4, M 3=574$ \\
\hline Alignments with Gblocks default & Harmonic Mean (LnL) & Bayes factor \\
\hline M1: mtDNA, nucDNA (2 partitions, 19 free parameters) & -27676.75 & $M 2, M 1=150.1$ \\
\hline M2: mtDNA, Wg, EF-1a, Bub3, RpS4, RpL27a (6 partitions, 59 parameters) & -27509.59 & $M 3, M 1=1210.5$ \\
\hline M3: mtDNA 1\&2, mtDNA 3, Wg, EF-1a, Bub3, RpS4, RpL27a (7 partitions, 69 parameters) & -26956.35 & $M 3, M 2=1060.4$ \\
\hline \multirow{3}{*}{$\begin{array}{l}\text { M4: mtDNA 1\&2, mtDNA 3, Wg 1\&2, Wg 3, EF-1a 1\&2, EF-1a 3, Bub3, RpS4, RpL27a } \\
\text { (9 partitions, } 89 \text { parameters) }\end{array}$} & -26691.65 & $M 4, M 1=1647.8$ \\
\hline & & $M 4, M 2=1497.73$ \\
\hline & & $M 4, M 3=437.3$ \\
\hline
\end{tabular}

the $\mathrm{A} / \mathrm{T}$ percentage in the $3 \mathrm{rd}$ codon positions in $\mathrm{Wg}$ and $E F-1 \alpha$ was only 32 and $45 \%$ respectively.

With the exception of $E F-1 \alpha$ 1st and 2nd codon positions $(18.9 \%)$, there was an overall higher rate of A-G and C-T transitions (from $60.8 \%$ for $R p L 27 a$ up to
91.6 \% for mtDNA 3rd codon positions). More precisely, mtDNA (all codon positions), Bub3 and $\mathrm{Wg}$ 1st \& 2nd codon positions were in excess of $\mathrm{C}$ - $\mathrm{T}$ transitions.

For protein-coding genes (mtDNA, $E F-1 \alpha$ and $W g$ ), the rate multiplier parameter $(\mathrm{m})$ was higher for the $3 \mathrm{rd}$

Table 4 Evolutionary properties of the partitions used in the study

\begin{tabular}{|c|c|c|c|c|c|c|}
\hline Partitions & $r(A \leftrightarrow C)$ & $r(A \leftrightarrow G)$ & $r(A \leftrightarrow T)$ & $r(C \leftrightarrow G)$ & $r(C \leftrightarrow T)$ & $r(G \leftrightarrow T)$ \\
\hline mtDNA $1 \& 2$ & $0.036(0.015-0.059)$ & $0.186(0.134-0.241)$ & $0.115(0.089-0.141)$ & $0.065(0.034-0.099)$ & $0.574(0.503-0.642)$ & $0.021(0.010-0.034)$ \\
\hline mtDNA 3 & $0.018(0.006-0.029)$ & $0.378(0.310-0.445)$ & $0.011(0.00-0.014)$ & $0.020(0.00-0.048)$ & $0.537(0.464-0.608)$ & $0.032(0.021-0.046)$ \\
\hline Wg $1 \& 2$ & $0.083(0.021-0.149)$ & $0.142(0.057-0.240)$ & $0.031(0.000-0.079)$ & $0.026(0.000-0.064)$ & $0.698(0.565-0.827)$ & $0.018(0.000-0.056)$ \\
\hline Wg 3 & $0.070(0.042-0.100)$ & $0.364(0.274-0.459)$ & $0.119(0.072-0.171)$ & $0.041(0.024-0.058)$ & $0.392(0.300-0.484)$ & $0.012(0.000-0.029)$ \\
\hline$E F-1 a 1 \& 2$ & $0.075(0 .-0.177)$ & $0.070(0.000-0.167)$ & $0.040(0-0.118)$ & $0.182(0.037-0.351)$ & $0.197(0.041-0.374)$ & $0.432(0.216-0.646)$ \\
\hline$E F-1 a 3$ & $0.052(0.025-0.082)$ & $0.481(0.373-0.588)$ & $0.072(0.031-0.120)$ & $0.018(0.004-0.035)$ & $0.342(0.243-0.438)$ & $0.031(0.008-0.059)$ \\
\hline Bub & $0.084(0.051-0.121)$ & $0.289(0.220-0.363)$ & $0.069(0.048-0.091)$ & $0.036(0.004-0.072)$ & $0.456(0.377-0.538)$ & $0.062(0.036-0.090)$ \\
\hline RpS4 & $0.068(0.047-0.090)$ & $0.341(0.296-0.388)$ & $0.104(0.085-0.123)$ & $0.070(0.042-0.099)$ & $0.332(0.288-0.378)$ & $0.082(0.062-0.104)$ \\
\hline RpL27a & $0.094(0.070-0.119)$ & $0.302(0.257-0.348)$ & $0.085(0.070-0.101)$ & $0.094(0.054-0.138)$ & $0.307(0.260-0.353)$ & $0.115(0.089-0.141)$ \\
\hline Partitions & pi A & pi $C$ & pi G & pi T & a (Shape parameter) & m (Rtae multiplier) \\
\hline mtDNA $1 \& 2$ & $0.271(0.242-0.299)$ & $0.137(0.120-0.155)$ & $0.147(0.124-0.171)$ & $0.443(0.414-0.472)$ & $0.133(0.118-0.148)$ & $0.580(0.483-0.681)$ \\
\hline mtDNA 3 & $0.418(0.392-0.444)$ & $0.049(0.044-0.055)$ & $0.051(0.045-0.057)$ & $0.480(0.453-0.506)$ & $0.635(0.549-0.729)$ & $8.929(8.34-9.524)$ \\
\hline Wg $1 \& 2$ & $0.284(0.234-0.333)$ & $0.215(0.171-0.260)$ & $0.288(0.237-0.339)$ & $0.211(0.169-0.258)$ & $0.076(0-0.181)$ & $0.034(0.021-0.048)$ \\
\hline Wg 3 & $0.151(0.119-0.182)$ & $0.402(0.349-0.452)$ & $0.278(0.231-0.327)$ & $0.168(0.137-0.201)$ & $1.086(0.776-1.415)$ & $1.254(0.984-1.535)$ \\
\hline$E F-1 a 1 \& 2$ & $0.307(0.260-0.354)$ & $0.212(0.170-0.254)$ & $0.258(0.213-0.305)$ & $0.222(0.180-0.264)$ & $0.093(0-0.258)$ & $0.029(0.004-0.014)$ \\
\hline$-1 a 3$ & $0.178(0.135-0.223)$ & $0.373(0.315-0.427)$ & $0.176(0.132-0.222)$ & $0.270(0.223-0.320)$ & $0.769(0.508-1.038)$ & $0.336(0.257-0.415)$ \\
\hline Bub & $0.351(0.314-0.387)$ & $0.129(0.105-0.153)$ & $0.169(0.141-0.197)$ & $0.349(0.313-0.385)$ & $0.222(0.166-0.279)$ & $0.190(0.152-0.229)$ \\
\hline RpS4 & $0.332(0.308-0.354)$ & $0.162(0.146-0.180)$ & $0.147(0.131-0.163)$ & $0.357(0.334-0.380)$ & $0.427(0.364-0.496)$ & $0.262(0.224-0.303)$ \\
\hline RpL27a & $0.390(0.367-0.412)$ & $0.109(0.096-0.123)$ & $0.111(0.097-0.124)$ & $0.389(0.366-0.410)$ & $0.820(0.693-0.946)$ & $0.536(0.455-0.619)$ \\
\hline
\end{tabular}




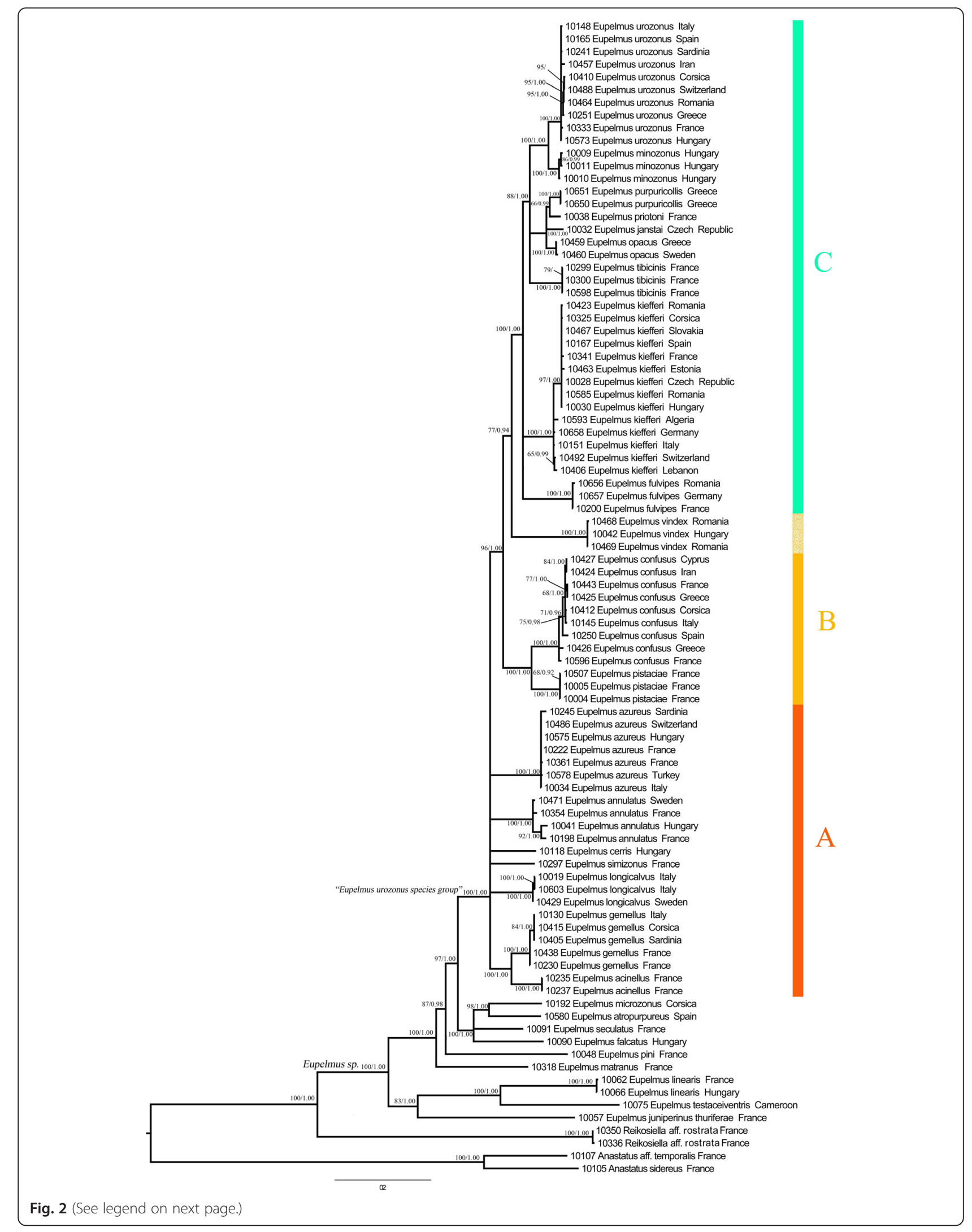


(See figure on previous page.)

Fig. 2 Phylogram of relationships among species of the "Eupelmus urozonus species group" obtained from the concatenated dataset alignment (3197 bp and 9 partitions) with Gblocks-default parameters. Uppercase letters refer to clades discussed in the text. Nodes with likelihood bootstrap (BP) values $<65$ have been collapsed. BP $(\geq 65)$ and Bayesian posterior probabilities $(\geq 0.90)$ are indicated at nodes. Each line represents a sequenced individual with information in the following order: molecular code, species, and country

codon positions. Thus, mtDNA 3rd codon positions evolved more than sixteen times faster than the fastest nuclear gene (RpL27a).

The shape parameter of the gamma distribution $(\alpha)$ was also higher for the 3rd codon position of the protein coding genes, indicating that these positions show lower rate heterogeneity among sites. Additionally, $\alpha$ was lower for $B u b 3$ than for $R p S 4$ and $R p L 27 a$, indicating that $B u b 3$ had a greater rate of heterogeneity among sites.

\section{Phylogenetic trees inferred from concatenated datasets Impacts of alignment strategy and reconstruction methods} ML and Bayesian topologies obtained from the concatenated alignments without Gblocks cleaning were more resolved than those obtained with removal of poorly aligned blocks. Whatever the partitioning scheme and regardless of whether or not divergent blocks were included in the analyses, most internal nodes were nevertheless statistically supported (BP value $\geq 65$, PP value $\geq 90$ ). Moreover, the 18 species recently defined by $\mathrm{Al}$ khatib et al. [32] and E. vindex were recovered as a monophyletic group.

Overall, topologies showed three major clades (A, B, C) that emerge on highly supported basal nodes (Figs. 1 and 2 and Additional file 2: Figures S1-S8). Three topological conflicts were observed depending on whether or not the Gblocks cleaning step was performed: (i) Clade A was not supported in topologies inferred from the datasets cleaned using Gblocks (Fig. 2 and Additional file 2: Figures S5-S8); (ii) E. vindex was sister to the rest of clade $\mathrm{C}$ in the topologies inferred from data sets cleaned using Gblocks (Fig. 2 and Additional file 2: Figures S5-S8), while it was sister to E. confusus and E. pistaciae (clade B) without Gblocks cleaning (Fig. 1 and Additional file 2: Figures $\mathrm{S} 1-\mathrm{S} 4$ ); (iii) the relationships of $E$. matranus and E. pini were resolved when Gblocks was used ( $\mathrm{PP}=1$ and 0.98 respectively) (Fig. 2 and Additional file 2: Figures S5-S8), but not resolved without Gblocks cleaning of data sets (Fig. 1 and Additional file 2: Figures S1-S4). Taken as a whole, we decided to favour the alignment without the Gblocks procedure for the comparative analysis in order to favour the resolution for the terminal nodes.

\section{Molecular relationships within the "Eupelmus urozonus species group"}

ML and Bayesian analyses performed on the most complex partitioning scheme without Gblocks cleaning produced similar topologies with only a few differences for poorly supported nodes (Additional file 2: Figure S1). We therefore mapped all node support values (BP \& PP) on the ML topology (Fig. 1).

In all analyses, the "E. urozonus species group" was recovered as monophyletic (Fig. 1) with a strong support. The group was subdivided into three clades, "clades" being defined here as a statistically-supported basal divergence including several species:

- Clade A included E. acinellus, E. annulatus, E. azureus, E. cerris, E. gemellus, E. longicalvus and E. simizonus, whose relative positions were not resolved to the exception of the sister species relationship between E. acinellus and E. gemellus $(\mathrm{BP}=100, \mathrm{PP}=1)$.

- Clade B included three species with $E$. vindex being sister to $E$. confusus plus $E$. pistaciae with strong support $(\mathrm{BP}=92, \mathrm{PP}=1)$.

- Clade $C$ included the remaining species and namely E. fulvipes, E. janstai, E. kiefferi, E. minozonus, E. opacus, E. priotoni, E. purpuricollis, E. tibicinis and E. urozonus. Within clade C, two well-supported (in each case, $\mathrm{BP}=100, \mathrm{PP}=1$ ) subclades-"sub-clade" being defined as a more terminal divergence including at least 2 species-can be distinguished (i) $E$. opacus, E. priotoni, E. purpuricollis and E. janstai; (ii) E. minozonus and E. urozonus. These two subclades together with E. tibicinis, whose exact phylogenetic position remains unclear, form a well-supported monophyletic group $(\mathrm{BP}=98, \mathrm{PP}=1)$.

\section{Comparative analysis and host uses}

There were significant interspecific differences for both the absolute (AOS-Kruskal-Wallis test: $\chi_{16 \mathrm{df}}^{2}=93.7$; $p<10^{-3}$; E. priotoni and E. simizonus discarded because of lack of replicates) and relative (ROS-Kruskal-Wallis test: $\chi_{16 \mathrm{df}}^{2}=109.2 ; p<10^{-3} ;$ E. priotoni and E. simizonus also discarded) ovipositor lengths (Fig. 3a). AOS ranged from $398 \mu \mathrm{m}$ in E. minozonus to a maximum of $1179 \mu \mathrm{m}$ in $E$. cerris while ROS ranged from a minimum of 0.58 in $E$. fulvipes to a maximum of 1.16 in E. janstai. Even if AOS and ROS medians were significantly correlated one with another (Kendall's rank correlation: $z=2.73 ; p=0.006$ ), some discrepancies were observed as for $E$ cerris which exhibits the highest AOS but an intermediate ROS (Fig. 3a).

Within the "Eupelmus urozonus species group", there was no significant correlation between similarity in ovipositor length and phylogenetic distance (Mantel test for 


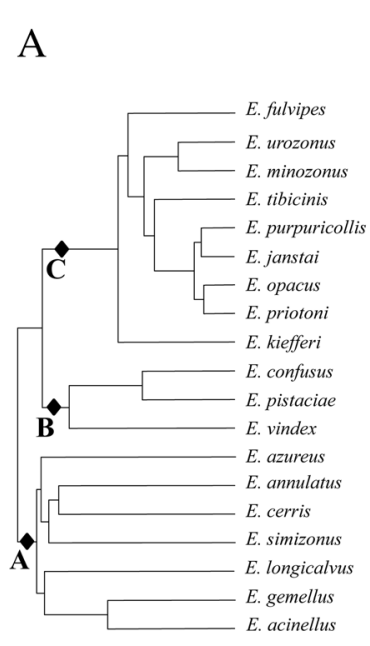

AOS

ROS

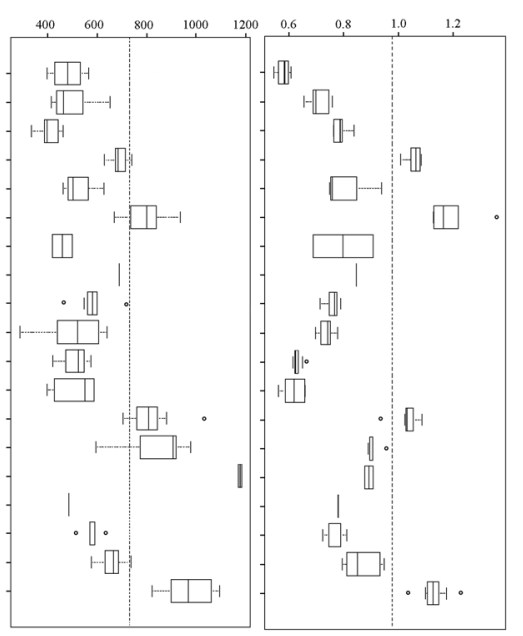

B

$\overbrace{\mathrm{Cec}}^{\mathrm{Col}} \overbrace{\mathrm{Cy}}^{\mathrm{Hip}} \mathrm{Hem} \quad$ Lep Neu

E. fulvipes

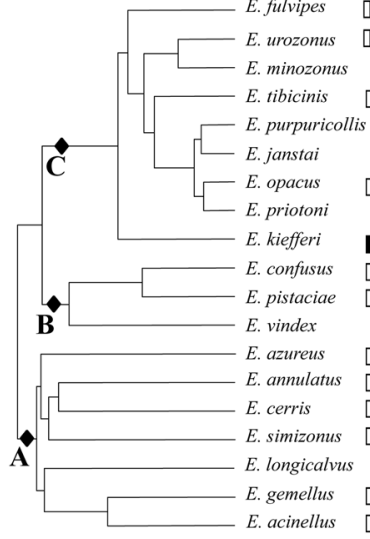

mسm

mाm

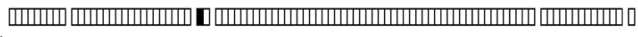

पाmm

I I I Im

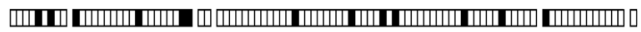

एسm

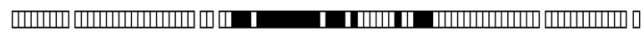

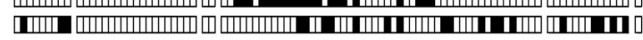

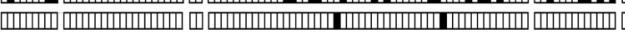

.

ए سm

एسmس

C

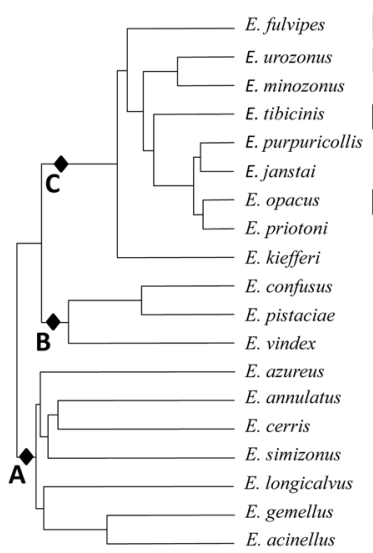

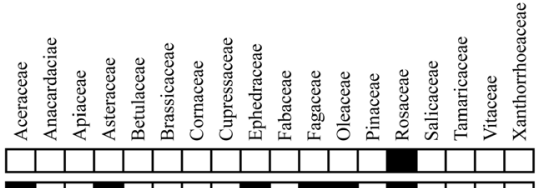

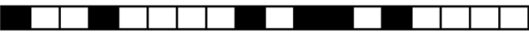

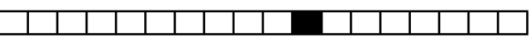

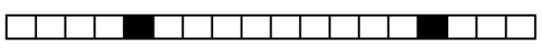
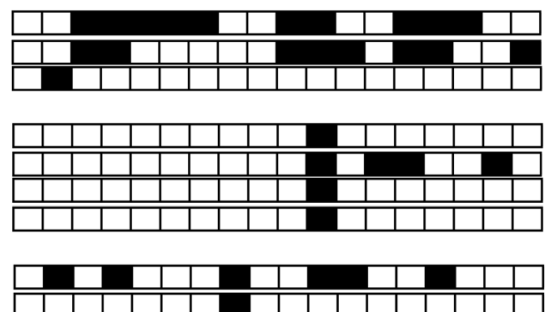

Fig. 3 (See legend on next page.) 
(See figure on previous page.)

Fig. 3 Mapping of ovipositor size and host ranges (host insect and related plants) along the multi-locus phylogeny of the "Eupelmus urozonus species group". The phylogenetic tree used is derived from the Fig. 1. For convenience, sizes of branches were modified but the topology remains unchanged. In Fig. 3a, boxplots are shown for the absolute (AOS in $\mu \mathrm{m}$ ) and relative (ROS - no unit) lengths of the ovipositor for each Eupelmus species. In each case, the vertical dotted line separates "short" versus "long" ovipositors. In Fig. 3b, the host specificity is indicated at three levels (from up to down): order, family, and species. Each rectangle indicates a possible host and the black ones indicate that at least one Eupelmus specimen was obtained from this host. In Fig. 3c, the plant host is indicated at the family level

AOS: $r=0.09, p=0.39-$ Mantel test for ROS: $r=0.08$, $p=0.44)$. When ovipositor length was treated as a binary variable with "long" ovipositors being those above the third quartile (4 or 5 cases among the 19 species), the observed D-statistics for AOS (0.13) and ROS (1.33) never departed from a random distribution (respectively $p=0.13$ and $p=0.61$ ) or a Brownian one (respectively $p=0.48$ and $p=0.14$ ). Consequently, it seems that no strong clustering existed on the length of the ovipositor sheaths. Remarkable differences in the length of the ovipositor sheaths were even observed between some sister species: $E$. acinellus-E. gemellus in clade $\mathrm{A}$ and $E$. janstai-E. purpuricollis in clade B (Fig. 3a).

Taken as a whole, our results indicated that both Cynipidae and Cecidomyiidae constitute the main host species for West Palearctic "E. urozonus species group" (Fig. 3b). Yet, contrasted feeding regimes (specialists versus generalists) were observed (Fig. 3b). Only three (E. acinellus, E. pistaciae and E. tibicinis) of the 13 species are strict specialists, with a distribution $(D=2.38)$ not significantly departing from both a random $(p=0.79)$ or a Brownian distribution $(p=0.11)$. At the family and order level (same distributions), three other species were specialists of Cynipidae-E. azureus (reported on 21 host species), E. cerris (2 hosts) and E. fulvipes (4 hosts)-and one (E. opacus) on Cecidomyiidae. At these levels, the relative distribution of specialists and generalists $(D=1.65)$ does not differs from a random $(p=0.72)$ or Brownian distribution $(p=0.10)$ and, as shown in Fig. 33b, about $50-60 \%$ of the described species in each of the three clades were specialists. The absence of a phylogenetic signal still holds under the assumption that all rare species (E. janstai, E. longicalvus, E. minozonus, E. priotoni, E. purpuricollis, $E$. vindex) are specialists. Departures from a random distribution is never significant (host species' level: $D=1.04$ with $p=0.51$ - host order's level: $D=1.52$ with $p=0.76$ ) while a significant departure is observed from a Brownian distribution at the host order's level (host species' level: $p=0.12$ - host order's level: $p=0.031)$. Interestingly, contrasted host ranges were observed between sister species: E. gemellus (six host species distributed in 3 orders) $-E$. acinellus (one host species) within clade A and E. confusus (thirteen species distributed in four orders) $-E$. pistaciae (one host species) within clade B (Fig. 3b).

We investigated the ability of the "E. urozonus species group" to parasitize host species belonging to Coleoptera,
Diptera, Hymenoptera and Lepidoptera (ordinal level) or Cecidomyiidae within Diptera and Cynipidae within Hymenoptera (familial level) (see Fig. 3b). However, in all these cases, we were not able to observe significant departures from a random or a Brownian distribution (See Additional file 3: Table S4).

Correlations between phylogenetic, morphometric (absolute or relative lengths of the ovipositor sheaths, AOS and ROS) and ecological (host ranges) matrices were also tested using simple or partial Mantel tests, at each of the three levels (species, family and order). Overall, the Mantel coefficients ranged between -0.07 and +0.14 and were never significantly different from zero (see Additional file 4: Table S3). At the host species level, such a result could be explained by the fact that only $24 \%$ of the hosts (mostly Cynipidae) are shared by at least two species of the "E. urozonus species group". As a consequence, this level of investigation may be too precise to detect any signal. However, such a limit cannot be taken into account at the two other taxonomic levels since about half of the host families and all host orders except Neuroptera are shared by at least two species of Eupelmus. Taken as a whole, these results confirm those obtained using D-statistics about the absence of significant phylogenetic constraints on the host range evolution. The relative ovipositor length also does not appear to be a significant driver of the host use.

When host plants rather than host insects are taken into account, 18 plant families were identified (see Fig. 3c), eight of which being used by only one Eupelmus species. However, four main families were used by at least four Eupelmus species: Asteraceae (4 species), Fagaceae (9 species), Rosaceae (5 species) and Salicaceae (4 species). For each of these families, no phylogenetic signal was detected using the D-statistics (See Additional file 3: Table S4). Additionally, no correlation was found between the related ecological matrix and the phylogenetic, and/or morphometric (AOS/ROS) matrices (see Additional file 5: Table S5).

\section{Discussion}

Phylogenetic relationships within the "E. urozonus species group"

Phylogenetic inter-specific relationships within the " $E$. urozonus species group" occurring in the Palaearctic region were recently investigated by $\mathrm{Al}$ khatib et al. [32] 
based on morphological characters and two genetic markers (mitochondrial COI and nuclear $\mathrm{Wg}$ ). This study showed an unsuspected diversity but it (i) failed to resolve phylogenetic relationships at both deep and intermediate levels, (ii) highlighted some discrepancies among tree topologies at the shallowest nodes resulting from COI and $\mathrm{Wg}$ sequences, (iii) did not include morphologically divergent but potentially phylogenetically closely related species. By considering new species and adding more informative markers, the present study improved the knowledge on the evolutionary history of the " $E$. urozonus species group".

Although the phylogenetic resolution was proven to be sensitive to inclusion or exclusion of divergent blocks by using Gblocks procedure from the sequence alignments, we obtained a reliable phylogeny which strongly supported the monophyly of our focus group of Eupelmus, including the 18 species treated in $\mathrm{Al}$ khatib et al. [32] and E. vindex, which is morphologically distinct from other members of the group in the shape of the syntergum and the anterior displacement of the ovipositor sheaths (Gibson \& Fusu, in prep). Additionally, the included species of the "E. urozonus species group" were distributed in three strongly supported clades, referred here as A, B and C (Fig. 1).

The molecular monophyly of the Palaearctic "E. urozonus species group" reflected in our concatenated datasets can be also supported through morphology. Al khatib et al. (in prep.) recently compared and combined the results of phylogenetic inferences using the molecular data presented here with morphological data. The main conclusion of this complementary work seems to be the structuration of Eupelmus as a set of independent species groups (including our focus group). Their delineation and their morphological supports are therefore not detailed here.

Despite using several loci from both the nuclear and mitochondrial genomes, some of the focal taxa remain poorly resolved. We expect that newer methods that dramatically increase the number of loci will help to better resolve these relationships (see for instance [78]).

\section{Ecological differentiation within the "E. urozonus species group"}

The diversification of parasitic organisms has been explained by various processes linking ecological specialization and speciation. For parasitoids, phylogenetic information and reliable host ranges are necessary to describe the patterns (distribution of generalist and specialist species) and to understand the underlying processes (e.g. "musical chairs" versus "oscillation"). This motivated the present work. Although members of the genus Eupelmus are usually described as generalist ectoparasitoids $[27,28]$, our study nevertheless leads to a more complex pattern. Our results indeed showed the coexistence of "strict" specialists restricted to one specific host (i.e. $E$ acinellus, E. pistaciae, E. tibicinis), intermediate specialists that can parasitize various species of Cynipidae (i.e. $E$. azureus, E. cerris and E. fulvipes) and generalists that are able to successfully develop on different insect orders (i.e. E. annulatus, E. confusus, E. gemellus and E. kiefferi).

This diversity in host use observed in the "E. urozonus species group" does not seem to be driven by phylogenetic history as generalists and specialists were recovered in each of the three clades. Moreover, some sister species exhibited fully contrasted ecologies (generalist species cited first): $E$. confusus $-E$. pistaciae and E. gemellus $-E$. acinellus. In this last case, because the facultative hyperparasitism lifestyle is recorded for some species of Eupelmus, we strongly suspect that E. gemellus develops as a hyperparasitoid of E. acinellus on Mesophleps oxycedrella (Lepidoptera). If this is true, it would mean that none of these generalists (E. confusus and E. gemellus) share any hosts with its sister species. Even if it is not the case, such contrasting patterns of host use remain, to our knowledge, rare in parasitoid species.

Quite similar conclusions arose when host plants instead hosts insects were taken into account. There was indeed no correlation between host plant ranges, phylogenetic and/or morphometric constraints. Moreover, the use of the four main plant families (Asteraceae, Fagaceae, Rosaceae and Salicaceae) did not seem to be constrained by the phylogenetic history. The underlying rationale of this complementary analysis was that host plants could at least partly determine ecological specialization of Eupelmus species insofar as the parasitoid species could use, innately or through learning, plant-linked cues in order to locate favourable environments, be the cues emitted passively (olfactory or visual information) or actively (synomones) (see for instance [79-81]). One criticism to this approach would, of course, be the level (plant family) at which our analysis was performed since it implies that only well-conserved cues could be detected.

A final facet of our investigation was the potential role of the ovipositor sheaths (as a proxy of the ovipositor length) as a driver of host use. The rationale was that (i) ovipositor structure could be constrained by the phylogenetic history of the species and, (ii) ovipositor length could determine accessibility to different hosts $[82,83]$. None of these hypotheses was however verified, ovipositor length appearing to be a very labile trait within our focus group.

Another driver of host range evolution could be the complexity of gall communities exploited by the Eupelmus species. Indeed, in numerous cases, Eupelmus species are occurring with numerous parasitoid species belonging to different chalcid families (e.g. Torymidae, Eurytomidae or Pteromalidae) which seem to be more functionally 
adapted to their hosts (see for instance [34, 84] and [85]). Such recurrent interspecific competitions may represent a potential limit for the abundance of Eupelmus but may also, ultimately, offer evolutionary opportunities. In particular, such an ecological intimacy could promote some switches towards unusual but ecologically related host insects and/or transitions towards other developmental modes (hyperparasitism or even predation). Such kind of adaptations may be illustrated by E. tibicinis, a specialist predator of the eggs of the red cicada, Tibicina haematodes (Scopoli, 1763) (Hemiptera: Tibicinidae).

\section{Conclusions}

This paper provides comprehensive information about the ecological differentiation within the Palaearctic species of the "E. urozonus species group" and contributes to our understanding of ecological specialization in parasitoids. Although further investigations are required, the intimate mixing of generalist and specialist species along the phylogeny leans toward the "oscillation hypothesis" (sensu Hardy and Otto [21]). It also raises new questions at both the inter- and intra-specific levels. At the intra-specific level, more detailed population genetics studies would be useful to test the existence of "host races" within generalist species, which could be a way to, (i) explain the capacity of a single species to develop in different hosts and (ii) offer opportunities for the recurrent apparition of specialized lineages and ultimately species. At the interspecific level, the partitioning of the available resources within sympatric Eupelmus species and with other chalcid wasps remains unclear. This would probably require a better knowledge of potential and realised host ranges, interspecific interactions (e.g., competition and hyperparasitism) and investigations on the influence of host plants on the associated parasitoids (e.g., attraction/repellence; phenology and structure of galls). Finally, an agronomic output of such investigations would be a better knowledge of the actual potential of some Eupelmus species to regulate certain insect pests such as the olive fruit fly, Bactrocera oleae (Gmelin, 1790) [86-89] or the chestnut gall wasp Dryocosmus kuriphilus Yasumatsu, 1951 [90-92].

\section{Availability of supporting data}

The data sets supporting the results are available in Dryad (doi: 10.5061/dryad.115m1).

All sequences are available in Genbank (http:// www.ncbi.nlm.nih.gov/genbank). Genbank accession numbers are given in Additional file 2: Table S2.

\section{Additional files}

Additional file 1: Table S1. Primer sequences used in the study and related references. Table S2. Information (including identification codes, taxonomic identity and Genbank accession numbers) related the specimens used in the phylogenetic analyses. (DOCX $50 \mathrm{~kb}$ )

Additional file 2: Figure S1. Trees from a) the $M L$ and b) Bayesian analyses of the combined dataset (without Gblocks cleaning, 9 partitions) Likelihood bootstrap values and posterior probabilities are indicated at nodes. Figure S2. Trees from a) the ML and b) Bayesian analyses of the combined dataset (without Gblocks cleaning, 7 partitions). Likelihood bootstrap values and posterior probabilities are indicated at nodes. Figure S3. Trees from a) the ML and b) Bayesian analyses of the combined dataset (without Gblocks cleaning, 6 partitions). Likelihood bootstrap values and posterior probabilities are indicated at nodes. Figure S4. Trees from a) the ML and b) Bayesian analyses of the combined dataset (without Gblocks cleaning, 2 partitions). Likelihood bootstrap values and posterior probabilities are indicated at nodes. Figure S5. Trees from a) the ML and b) Bayesian analyses of the combined dataset (with Gblocks-default parameters, 9 partitions). Likelihood bootstrap values and posterior probabilities are indicated at nodes. Figure S6. Trees from a) the ML and b) Bayesian analyses of the combined dataset (with Gblocks-default parameters, 7 partitions). Likelihood bootstrap values and posterior probabilities are indicated at nodes. Figure S7. Trees from a) the ML and b) Bayesian analyses of the combined dataset (with Gblocks-default parameters, 6 partitions). Likelihood bootstrap values and posterior probabilities are indicated at nodes. Figure S8. Trees from a) the ML and b) Bayesian analyses of the combined dataset (with Gblocks-default parameters, 2 partitions). Likelihood bootstrap values and posterior probabilities are indicated at nodes. Figure S9. Tree from the ML analysis of the mitochondrial partition. Likelihood bootstrap values (1000 replicates) and posterior probabilities are indicated at nodes. Figure S10. Tree from the $\mathrm{ML}$ analysis of the $\mathrm{Wg}$ locus. Likelihood bootstrap values (1000 replicates) and posterior probabilities are indicated at nodes. Figure S11. Tree from the ML analysis of the EF-1a locus. Likelihood bootstrap values (1000 replicates) and posterior probabilities are indicated at nodes. Figure S12. Tree from the ML analysis of the Bub3 locus (without Gblocks cleaning). Likelihood bootstrap values (1000 replicates) and posterior probabilities are indicated at nodes. Figure S13. Tree from the ML analysis of the Bub3 locus (with Gblocks-default parameters). Likelihood bootstrap values (1000 replicates) and posterior probabilities are indicated at nodes. Figure S14. Tree from the ML analysis of the RpS4 locus (without Gblocks cleaning). Likelihood bootstrap values (1000 replicates) and posterior probabilities are indicated at nodes. Figure S15. Tree from the $\mathrm{ML}$ analysis of the RpS4 locus (with Gblocks-default parameters). Likelihood bootstrap values (1000 replicates) and posterior probabilities are indicated at nodes. Figure S16. Tree from the ML analysis of the RpL27a locus (without Gblocks cleaning). Likelihood bootstrap values (1000 replicates) and posterior probabilities are indicated at nodes. Figure S17. Tree from the ML analysis of the RpL27a locus (with Gblocks-default parameters). Likelihood bootstrap values (1000 replicates) and posterior probabilities are indicated at nodes. Figure S18. Illustrations of morphometric measurements on Eupelmus females. (A) ovipositor sheaths, (B) ovipositor stylet (second and third pairs of valvulae), and (C) hind tibia. (PDF $110496 \mathrm{~kb}$ )

Additional file 3: Table S4. Summary of information related to the detection of a phylogenetic signal (both host insects and plants). (DOCX $20 \mathrm{~kb}$ )

Additional file 4: Table S3. Summary of Mantel tests used for the comparative analysis dealing with host insects. (DOCX $21 \mathrm{~kb}$ )

Additional file 5: Table S5. Summary of Mantel tests used for the comparative analysis dealing with host plants. (DOCX 19 kb)

\section{Competing interests}

The authors declare that they have no competing interests.

\section{Authors' contributions}

AC, FAK, GD, LF, JYR and NR conceived the study. FAK, GD, LF and NR provided the biological material and related information. AC, FAK and GG performed the molecular characterization. FAK, GD and LF realised the morphological measurements. AC, FAK, GD and NR realised the analysis. FAK, GD and NR drafted the manuscript with input from the other authors. All authors read and approved the final manuscript. 


\section{Acknowledgements}

This research was directly supported by the projects "EUPELMUS" (Scientific Department "Santé des Plantes et Environnement" of INRA—grant 2012-04-05-20), "INULA" (APR Pesticides 2011: "Changer les pratiques agricoles pour préserver les services écosystémiques") and 'BIOFIS' (number 1001-001) allocated by the French Agropolis Fondation (Montpellier). This project was supported by the network Bibliothèque du Vivant funded by the CNRS, the Muséum National d'Histoire Naturelle (MNHN) and the Institut National de la Recherche Agronomique (INRA), and technically supported by the Genoscope. This study also took benefits from field work conducted in the frame of the projects "BIOINV-4I" (ANR-06-BDIV-008 - Coordinator: Thomas Guillemaud), "Lutte biologique contre le Cynips du Châtaignier" (ONEMA 2011 - Coordinator: Nicolas Borowiec \& Jean-Claude Malausa), and the Swedish Malaise Trap Project (SMTP).

The authors want to thank Sylvie Warot for her contribution to the molecular work as well as two anonymous reviewers who helped to improve the first draft.

\section{Author details}

'INRA, University of Nice Sophia Antipolis, CNRS, UMR 1355-7254 Institut Sophia Agrobiotech, Sophia Antipolis 06900, France. ${ }^{2}$ INRA, UMR 1062 CBGP, 755 avenue du Campus Agropolis, CS 30016 F-34988, Montferrier-sur-Lez Cedex, France. ${ }^{3}$ CIRAD, UMR 55 CBGP, 755 Avenue du Campus Agropolis, CS 30016 F-34988, Montferrier-sur-Lez Cedex, France. ${ }^{4}$ Faculty of Biology, Alexandru loan Cuza University, Bd. Carol I nr. 11, 700506 lasi, Romania.

Received: 3 July 2015 Accepted: 15 December 2015

Published online: 19 January 2016

\section{References}

1. Matsubayashi KW, Ohshima I, Nosil P. Ecological speciation in phytophagous insects. Entomol Exp Appl. 2010;134:1-27.

2. Rundle HD, Nosil P. Ecological speciation. Ecol Lett. 2005:8:336-52.

3. Schluter D. Ecology and the origin of species. Trends Ecol Evol. 2001;16:372-80.

4. Schluter D. Evidence for ecological speciation and its alternative. Science. 2009;323:737-41.

5. Futuyma DJ. Sympatric speciation: norm or exception. In: Tilmon KJ, editor Specialization, speciation and radiation: the evolutionary biology of herbivorous insects. Berkeley: University of California Press; 2008. p. 136-48.

6. Nyman T, Vikberg V, Smith DR, Boeve JL. How common is ecological speciation in plant-feeding insects? A 'Higher' Nematinae perspective. BMC Evol Biol. 2010;10:266

7. Feder $J$, Forbes AA. Sequential speciation and the diversity of parasitic insects. Ecol Entomol. 2010;35:67-76.

8. Forbes AA, Powell THQ, Stelinski LL, Smith JJ, Feder JL. Sequential sympatric speciation across trophic levels. Science. 2009;323:776-9.

9. Stireman JO, Nason JD, Heard SB, Seehawer JM. Cascading host-associated genetic differentiation in parasitoids of phytophagous insects. Proc R Soc B-Biol Sci. 2006;273:523-30.

10. Armbruster WS, Baldwin BG. Switch from specialized to generalized pollination Nature. 1998;394:632.

11. Moran NA. The evolution of host-plant alternation in aphids: evidence for specialization as a dead end. Am Nat. 1988;132:681-706.

12. Nosil P. Transition rates between specialization and generalization in phytophagous insects. Evolution. 2002;56:1701-6.

13. Vamosi JC, Armbruster WS, Renner SS. Evolutionary ecology of specialization: insights from phylogenetic analysis. Proc R Soc B-Biol Sci. 2014;281:20142004.

14. Futuyma DJ, Moreno G. The evolution of ecological specialization. Annu Rev Ecol Syst. 1988;19:207-33.

15. Jaenike J. Host specialization in phytophagous insects. Annu Rev Ecol Syst. 1990;21:243-73

16. Via S. Ecological genetics and host adaptation in herbivorous insects: the experimental study of evolution in natural and agricultural systems. Annu Rev Entomol. 1990:35:421-46.

17. Carletto J, Lombaert E, Chavigny P, Brevault T, Lapchin L, VanlerbergheMasutti F. Ecological specialization of the aphid Aphis gossypii Glover on cultivated host plants. Mol Ecol. 2009;18:2198-212.

18. De Barro PJ. Genetic structure of the whitefly Bemisia tabaci in the Asia-Pacific region revealed using microsatellite markers. Mol Ecol. 2005;14:3695-718.
19. Frantz A, Plantegenest M, Mieuzet L, Simon JC. Ecological specialization correlates with genotypic differentiation in sympatric host-populations of the pea aphid. J Evol Biol. 2006;19:392-401.

20. Colles A, Liow LH, Prinzing A. Are specialists at risk under environmental change? Neoecological, paleoecological and phylogenetic approaches. Ecol Lett. 2009;12:849-63.

21. Hardy NB, Otto SP. Specialization and generalization in the diversification of phytophagous insects: tests of the musical chairs and oscillation hypotheses. Proc R Soc B-Biol Sci. 2014;281:20132960.

22. Godfray HCJ. Parasitoids: Behavioral and Evolutionary Ecology. Princeton: Princeton University Press; 1994.

23. Quicke DLJ, Fitton MG, Tunstead JR, Ingram SN, Gaitens PV. Ovipositor structure and relationships within the Hymenoptera, with special reference to the Ichneumonoidea. J Nat Hist. 1994;28:635-82.

24. Sivinski J, Aluja M. The evolution of ovipositor length in the parasitic Hymenoptera and the search for predictability in biological control. Fla Entomol. 2003;86:143-50.

25. Vilhelmsen L, Turrisi GF. Per arborem ad astra: Morphological adaptations to exploiting the woody habitat in the early evolution of Hymenoptera. Arthropod Struct Dev. 2011;40:2-20.

26. Noyes JS. Universal Chalcidoidea Database. 2015. http://www.nhm.ac.uk/ research-curation/research/projects/chalcidoids/index.html.

27. Gibson GAP. Parasitic wasps of the subfamily Eupelminae: classification and revision of world genera (Hymenoptera: Chalcidoidea, Eupelmidae). Mem Entomol Intl. 1995;5:i-v + 421.

28. Gibson GAP. Revision of the genus Macroneura Walker in America north of Mexico (Hymenoptera: Eupelmidae). Can Entomol. 1990;122:837-73.

29. Askew RR, Nieves-Aldrey JL. The genus Eupelmus Dalman, 1820 (Hymenoptera, Chalcidoidea, Eupelmidae) in peninsular Spain and the Canary Islands, with taxonomic notes and descriptions of new species. Graellsia. 2000;56:58-60.

30. Bouček Z. Australasian Chalcidoidea (Hymenoptera). A biosystematic revision of genera of fourteen families, with a reclassification of species. Aberystwyth: CAB International Institute of Entomology, the Cambrian News Ltd.; 1988.

31. Gibson GAP. The species of Eupelmus (Eupelmus) Dalman and Eupelmus (Episolindelia) Girault (Hymenoptera: Eupelmidae) in North America north of Mexico. Zootaxa. 2011:2951:1-97.

32. Al khatib F, Fusu L, Cruaud A, Gibson G, Borowiec N, Rasplus J-Y, et al. An integrative approach to species discrimination in the Eupelmus urozonus complex (Hymenoptera, Eupelmidae), with the description of 11 new species from the Western Palaearctic. Syst Entomol. 2014;39:806-62.

33. Al khatib F, Fusu L, Cruaud A, Gibson G, Borowiec N, Rasplus J-Y, et al. Availability of eleven species names of Eupelmus (Hymenoptera, Eupelmidae) proposed in Al khatib et al. (2014). Zookeys. 2015;505:137-45.

34. Kaartinen R, Stone GN, Hearn J, Lohse K, Roslin T. Revealing secret liaisons: DNA barcoding changes our understanding of food webs. Ecol Entomol. 2010;35:623-38.

35. Fusu L. A revision of the Palaearctic species of Reikosiella (Hirticauda) (Hymenoptera, Eupelmidae). Zootaxa. 2013;3636:01-34

36. Kalina V. The Palearctic species of the genus Macroneura Walker, 1837 (Hymenoptera, Chalcidoidea, Eupelmidae), with descriptions of new species. Sbornik Vedeckeho Lesnickeho Ustavu Vysoke Skoly Zemedelske v Praze. 1981;24:83-111.

37. Kalina V. The Palearctic species of the genus Anastatus Motschulsky, 1860 (Hymenoptera, Chalcidoidea, Eupelmidae), with descriptions of new species. Silvaecultura Tropica et Subtropica. 1981;8:3-25.

38. Cruaud A, Jabbour-Zahab R, Genson G, Cruaud C, Couloux A, Kjellberg F, et al. Laying the foundations for a new classification of Agaonidae (Hymenoptera: Chalcidoidea), a multilocus phylogenetic approach. Cladistics. 2010;26:359-87.

39. Cruaud A, Underhill JG, Huguin M, Genson G, Jabbour-Zahab R, Tolley KA, Rasplus J-Y, van Noort S. A multilocus phylogeny of the world Sycoecinae fig wasps (Chalcidoidea: Pteromalidae). PLoS One. 2013; 8(11):e79291. doi:10.1371/journal.pone.0079291.

40. Deuve T, Cruaud A, Genson G, Rasplus J-Y. Molecular systematics and evolutionary history of the genus Carabus (Col. Carabidae). Mol Phylogenet Evol. 2012;65:259-75.

41. Gomez-Zurita J, Cardoso A. Systematics of the New Caledonian endemic genus Taophila Heller (Coleoptera: Chrysomelidae, Eumolpinae) combining morphological, molecular and ecological data, with description of two new species. Syst Entomol. 2014;39:111-26. 
42. Brower AVZ, DeSalle R. Patterns of mitochondrial versus nuclear DNA sequence divergence among nymphalid butterflies: the utility of wingless as a source of characters for phylogenetic inference. Insect Mol Biol. 1998;7:73-82.

43. Campbell DL, Brower AVZ, Pierce NE. Molecular evolution of the wingless gene and its implications for the phylogenetic placement of the butterfly family Riodinidae (Lepidoptera : Papilionoidea). Mol Biol Evol. 2000;17:684-96.

44. Haine ER, Martin J, Cook JM. Deep mtDNA divergences indicate cryptic species in a fig-pollinating wasp. BMC Evol Biol. 2006;6:83.

45. Pilgrim EM, von Dohlen CD, Pitts JP. Molecular phylogenetics of Vespoidea indicate paraphyly of the superfamily and novel relationships of its component families and subfamilies. Zool Scr. 2008;37:539-60.

46. Rokas A, Nylander JAA, Ronquist F, Stone GN. A maximum-likelihood analysis of eight phylogenetic markers in gallwasps (Hymenoptera: Cynipidae): implications for insect phylogenetic studies. Mol Phylogenet Evol. 2002;22:206-19.

47. Belshaw R, Quicke DLJ. A molecular phylogeny of the Aphidiinae (Hymenoptera: Braconidae). Mol Phylogenet Evol. 1997;7:281-93.

48. Cho SW, Mitchell A, Regier JC, Mitter C, Poole RW, Friedlander TP, et al. A highly conserved nuclear gene for low-level phylogenetics: Elongation factor-1-alpha recovers morphology-based tree for heliothine moths. Mol Biol Evol. 1995;12:650-6.

49. Lin CP, Danforth BN. How do insect nuclear and mitochondrial gene substitution patterns differ? Insights from Bayesian analyses of combined datasets. Mol Phylogenet Evol. 2004;30:686-702.

50. Mitchell A, Cho S, Regier JC, Mitter C, Poole RW, Matthews M. Phylogenetic utility of elongation factor-1a in Noctuoidea (Insecta: Lepidoptera): the limits of synonymous substitution. Mol Biol Evol. 1997;14:381-90.

51. Mitchell A, Mitter C, Regier JC. More taxa or more characters revisited: combining data from nuclear protein-encoding genes for phylogenetic analyses of Noctuoidea (Insecta: Lepidoptera). Syst Biol. 2000;49:202-24.

52. Kawakita A, Ascher JS, Sota T, Kato M, Roubik DW. Phylogenetic analysis of the corbiculate bee tribes based on 12 nuclear protein-coding genes (Hymenoptera: Apoidea: Apidae). Apidologie. 2008;39:163-75.

53. Rasmussen C, Cameron SA. Global stingless bee phylogeny supports ancient divergence, vicariance, and long distance dispersal. Biol J Linn Soc. 2010;99:206-32.

54. Lohse K, Sharanowski B, Blaxter M, Nicholls JA, Stone GN. Developing EPIC markers for chalcidoid Hymenoptera from EST and genomic data. Mol Ecol Resour. 2011;11:521-9.

55. Lohse K, Sharanowski B, Stone GN. Quantifying the Pleistocene history of the oak gall parasitoid Cecidostiba fungosa using twenty intron loci. Evolution. 2010;64:2664-81.

56. Sharanowski BJ, Robbertse B, Walker J, Voss SR, Yoder R, Spatafora J, et al. Expressed sequence tags reveal Proctotrupomorpha (minus Chalcidoidea) as sister to Aculeata (Hymenoptera: Insecta). Mol Phylogenet Evol. 2010;57:101-12.

57. Edgar RC. MUSCLE: a multiple sequence alignment method with reduced time and space complexity. BMC Bioinformatics. 2004;5:1-19.

58. Gouy M, Guindon S, Gascuel O. SeaView version 4: a multiplatform graphical user interface for sequence alignment and phylogenetic tree building. Mol Biol Evol. 2010;27:221-4.

59. Castresana J. Selection of conserved blocks from multiple alignments for their use in phylogenetic analysis. Mol Biol Evol. 2000;17:540-52.

60. Tamura K, Peterson D, Peterson N, Stecher G, Nei M, Kumar S. MEGA5: molecular evolutionary genetics analysis using maximum likelihood, evolutionary distance, and maximum parsimony methods. Mol Biol Evol. 2011;28:2731-9.

61. Kass RE, Raftery AE. Bayes factors. J Am Stat Assoc. 1995;90(430):773-95.

62. Nylander JAA, Ronquist F, Huelsenbeck JP, Nieves-Aldrey JL. Bayesian phylogenetic analysis of combined data. Syst Biol. 2004;53:47-67.

63. Pagel M, Meade A. Mixture models in phylogenetic inferences. In: Gascuel O, editor. Mathematics of Evolution and Phylogeny. Oxford: Clarendon; 2005. p. 121-42.

64. Posada D. jModelTest: phylogenetic model averaging. Mol Biol Evol. 2008; 25:1253-6.

65. Stamatakis A. RAxML version 8: a tool for phylogenetic analysis and post-analysis of large phylogenies. Bioinformatics. 2014;30:1312-3.

66. Ronquist F, Teslenko M, van der Mark P, Ayres DL, Darling A, Hohna S, et al. MrBayes 3.2: efficient bayesian phylogenetic inference and model choice across a large model space. Syst Biol. 2012;61:539-42.

67. Geyer CJ. Markov chain Monte Carlo maximum likelihood. In Keramidas EM, Kaufman SM, editors. Computing Science and Statistics. Proceedings of the 23rd Symposium on the Interface. Fairfax: Interface Foundation of North America; 1991; p. 156-163.
68. Rambaut A, Suchard MA, Xie D, Drummond AJ. Tracer v1.6. 2014. http:// beast.bio.ed.ac.uk/Tracer.

69. Miller MA, Pfeiffer W, Schwartz T. Creating the CIPRES Science Gateway for inference of large phylogenetic trees. In: Gateway Computing Environments Workshop (GCE). New Orleans 2010; 1-8.

70. Fritz SA, Purvis A. Selectivity in mammalian extinction risk and threat types: a new measure of phylogenetic signal strength in binary traits. Conserv Biol. 2010;24:1042-51.

71. Legendre P, Fortin MJ. Comparison of the Mantel test and alternative approaches for detecting complex multivariate relationships in the spatial analysis of genetic data. Mol Ecol Resour. 2010;10:831-44.

72. Harmon LJ, Glor RE. Poor statistical performance of the Mantel test in phylogenetic comparative analyses. Evolution. 2010;64:2173-8.

73. Dray S, Dufour AB. The ade4 package: Implementing the duality diagram for ecologists. J Stat Softw. 2007;22:1-20.

74. Paradis E, Claude J, Strimmer K. APE: Analyses of Phylogenetics and Evolution in R language. Bioinformatics. 2004;20:289-90.

75. Goslee SC, Urban DL. The ecodist package for dissimilarity-based analysis of ecological data. J Stat Softw. 2007;22:1-19.

76. Oksanen JBF, Kindt R, Legendre P, Minchin PR, O'Hara RB, Simpson GL, Solymos P, Stevens MHH, Wagner H: "vegan" - Community ecology package. 2015. http:// vegan.r-forge.r-project.org/FAQ-vegan.html\#How-tocite-vegan_003f.

77. Yang ZH. Maximum likelihood phylogenetic estimation from DNA sequences with variable rates over sites: approximate methods. J Mol Evol. 1994;39:306-14.

78. Cruaud A, Gautier M, Galan M, Foucaud J, Saune L, Genson G, et al. Empirical assessment of RAD sequencing for interspecific phylogeny. Mol Biol Evol. 2014;31:1272-4.

79. Segura DF, Viscarret MM, Paladino LZC, Ovruski SM, Cladera JL. Role of visual information and learning in habitat selection by a generalist parasitoid foraging for concealed hosts. Anim Behav. 2007;74:131-42.

80. Turlings TCJ, Tumlinson JH, Eller FJ, Lewis WJ. Larval-damaged plants: source of volatile synomones that guide the parasitoid Cotesia marginiventris to the micro-habitat of its hosts. Entomol Exp Appl. 1991;58:75-82.

81. Vinson SB. The general host selection behavior of parasitoid Hymenoptera and a comparison of initial strategies utilized by larvaphagous and oophagous species. Biol Control. 1998;11:79-96.

82. Austin AD, Field SA. The ovipositor system of scelionid and platygastrid wasps (Hymenoptera: Platygastroidea): comparative morphology and phylogenetic implications. Invertebr Taxon. 1997;11:1-87.

83. Ghara M, Kundanati L, Borges RM. Nature's swiss army knives: ovipositor structure mirrors ecology in a multitrophic fig wasp community. PLoS One. 2011;6(8):e23642. doi:10.1371/journal.pone.0023642.

84. László Z, Tóthmérész B. Parasitism, phenology and sex ratio in galls of Diplolepis rosae in the Eastern Carpathian Basin. Entomologica Romanica. 2011;16:33-8.

85. Delanoue P. The consequence of competition between indigenous chalcidoids and an introduced braconid (Opius concolor) in attempts to limit populations of Dacus oleae in the Alpes-Maritimes. Revue de Pathologie Végétale et d'Entomologie Agricole de France. 1964;43:145-51.

86. Boccaccio L, Petacchi R. Landscape effects on the complex of Bactrocera oleae parasitoids and implications for conservation biological control. Biocontrol. 2009;54:607-16.

87. Borowiec N, Groussier-Bout G, Vercken E, Thaon M, Auguste-Maros A, WarotFricaux $S$, et al. Diversity and geographic distribution of the indigenous and exotic parasitoids of the olive fruit fly, Bactrocera oleae (Diptera: Tephritidae), in Southern France. IOBC-WPRS Bull. 2012;79:71-8.

88. Neuenschwander P, Bigler F, Delucchi V, Michelakis S. Natural enemies of preimaginal stages of Dacus oleae Gmel. (Dipt., Tephritidae) in Western Crete. I. Bionomics and phenologies. Bollettino del Laboratorio di Entomologia Agraria 'Filippo Silvestri'. 1983;40:3-32.

89. Warlop F. Limitation of olive pest populations through the development of conservation biocontrol. Cah Agric. 2006;15:449-55.

90. Aebi A, Schonrogge K, Melika G, Quacchia A, Alma A, Stone GN. Native and introduced parasitoids attacking the invasive chestnut gall wasp Dryocosmus kuriphilus. Bull OEPP/EPPO Bull. 2007;37:166-71.

91. Borowiec N, Thaon M, Brancaccio L, Warot S, Vercken E, Fauvergue X, et al. Classical biological control against the chestnut gall wasp Dryocosmus kuriphilus (Hymenoptera, Cynipidae) in France. Plant Prot Q. 2014;29:7-10.

92. Quacchia A, Ferracini C, Nicholls JA, Piazza E, Saladini MA, Tota F, et al. Chalcid parasitoid community associated with the invading pest Dryocosmus kuriphilus in north-western Italy. Insect Conserv Divers. 2013;6:114-23. 